\title{
Bounds on Outputs of the Exact Weak Solution of the Three-Dimensional Stokes problem
}

\author{
Zhong Cheng; Shahin Ghomeshi†, Marius Paraschivoiu ${ }^{\ddagger}$ \\ Department of Mechanical and Industrial Engineering, Concordia University
}

\begin{abstract}
A method for obtaining rigorous upper and lower bounds on an output of the exact weak solution of the three dimensional Stokes problem is described. Recently bounds for the exact outputs of interest have been obtained for both the Poisson equation and the advection-diffusion-reaction equation. In this work, we extend this approach to the Stokes problem where a novel formulation of the method also leads to a simpler flux calculation based on the directly equilibrated flux method. To illustrate this technique, bounds on the flowrate are calculated for an incompressible creeping flow driven by a pressure gradient in an endless square channel with an array of rectangular obstacles in the center.
\end{abstract}

\section{KEY WORDS}

Stokes problem, output bounds, exact weak solution, three dimensional space

\section{Introduction}

When performing computer simulations, the presence of numerical errors in the solution leads to uncertainty of the design quantities. In order to gain confidence in the prediction of these quantities, spacial convergence studies are often performed, which can become computationally very expensive, especially for three dimensional problems. For this reason, adaptive mesh strategies are often performed. However, one disadvantage of adaptive methods is that a desired error reduction target is not guaranteed. In order to obtain reliability in the numerical approximation, efficient error estimators are necessary, but the questions "what error is relevant" and "will an adaptive mesh refinement lead to a desired solution accuracy" must be answered.

Since the 1970s, error estimation based on either the a priori or a posteriori procedures have been developed. An overview of pioneer work on estimation of the global error such as the error in energy can be found in $[1,12]$. Latter work on the investigation of possible extensions of error norms [2] lead to the idea of error estimators associated with outputs (also termed goal oriented error estimation) which was further extended in the late 1990s [14, 18, 19, 23]. These procedures are motivated by engineering practice where the error must be related to quantities or "outputs of interest" which are functionals of field variables such as displacements, velocity field, or pressure. Initially applied to elliptic problems, including the Stokes problem $[20,13,16]$, but more recently, goal oriented error estimation techniques have been advanced in the study of transient parabolic problems [10] and also acoustic wave propagation problems [15].

More recently, guaranteed bounds on exact outputs also called "exact bounds" have been

\footnotetext{
*EMAIL: zcheng@encs. concordia.ca

$\dagger$ EMAIL: s_ghomes@encs. concordia.ca

$\ddagger$ EMAIL: corresponding author, paraschi@encs.concordia.ca
} 
proposed in $[24,25,21]$ and for time dependent outputs in [4]. The upper and lower computed bounds, hold for all levels of refinement and are shown to approach the exact quantity of interest at the same rate as its underlying finite element approach. This certificate of precision can then determine the "best" as well as the "worst" case scenario in an engineering design problem. Moreover the method can be termed "cost effective" as it can be used to determine the size of the mesh required to achieve a desired level of accuracy. This approach also answers the above question where error in a design quantity is important.

The strategy involved in the computing of bounds on exact outputs of interest is similar to the former hierarchical method [18], in that it involves decomposing the global mesh into several elemental subdomains and relaxing the continuity requirements along the edges of each subdomain. A Lagrangian is first constructed so that the output problem is recasted as a constrained minimization problem where the constraints are the continuity requirements along the edges of the subdomains and the equilibrium equation. The gradient condition of the Lagrangian will then lead to the primal-adjoint pair and the equilibration equation that will determine the candidate inter-element continuity multipliers. The bounds are finally obtained through local subproblem calculations. At this stage, the method differs from the former two-level residual method because by exploiting the Lagrangian saddle point property, existence of such bounds on the exact solution output is guaranteed, however the bounds are uncomputable in general. The key ingredient relies on constructing a complementary energy functional chosen from a suitable finite dimensional set that can be used to bound the infinite dimensional problem [24, 25].

Our goal in this paper is to extend the exact bound method for the Stokes problem based on the above implicit procedure. The extension of the bounds on exact outputs to the Stokes equations is similar to the Stokes hierarchical method [20] but aims at bounding the exact outputs. Similarly, the global primal-dual calculations derived from the minimization problems of the augmented Lagrangian with the relaxation of the continuity requirements along the edges of each subdomain and the primal-dual hybrid flux calculations for the inter-subdomain connectivities are solved. Then the computed solutions for the primal and dual problems (velocities, pressures and adjoints) and hybrid fluxes are interpolated from the low-order polynomial space (e.g. $\mathbf{P}^{1++}-\mathbf{P}^{1}$ [3] as shown in the left picture of Figure 1) to a higher order polynomial space (e.g. $\mathbf{P}^{2+}-\mathbf{P}^{1}$ $[9,3]$, as shown in the right of Figure 1). This process is different from the hierarchical method in that the interpolation can be performed on the same mesh as the global and hybrid flux calculations. Finally, the augmented Lagrangian is reformulated by implementing a complementary energy functional chosen from a suitable finite dimensional set that can be used to bound the infinite dimensional Stokes problem. This step is also different from the hierarchical method because the upper and lower bounds are computed by finding the inf-sup condition of the complimentary energy reformulated augmented Lagrangian on the decoupled subdomain so that they can bound the exact Stokes output.

To illustrate the exact bounds method for the Stokes equations, we select a benchmark problem for a creeping flow in a channel [5] and derive the mathematical formulation and bounds procedures as follows.

\section{Review of the Exact Bound Method}

\subsection{Model Problem: Poisson's equation}

For clarity, before extending to the Stokes equations, we first review the exact bound method presented in [24, 25, 21]. We consider Poisson's equation for a three dimensional cube geometry $(\Omega)$ with homogeneous Dirichlet boundaries, $\Gamma=\partial \Omega$ as it is the simplest model problem. The 

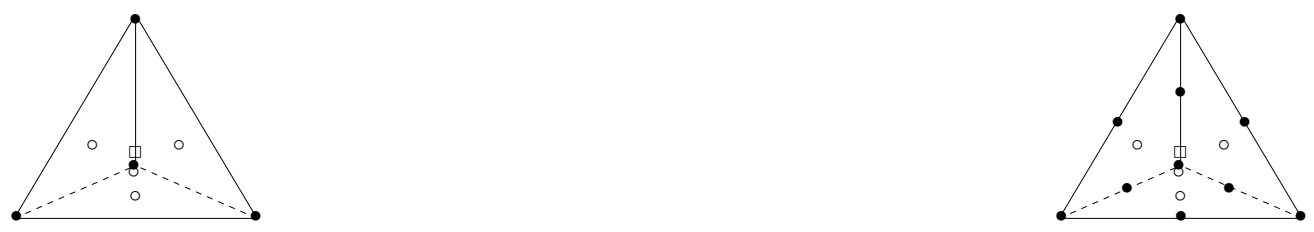

Figure 1: Tetrahedral elements for velocity field. • vertex nodes; o mid-face bubble nodes; centroid nodes. (Left: $\mathbf{P}^{1++}-\mathbf{P}^{1}[3]$; right: $\mathbf{P}^{2+}-\mathbf{P}^{1}[9,3]$ ).

formulation for the Poisson's problem is then stated weakly as: find $u \in \mathcal{U}$ such that

$$
\int_{\Omega} \nabla u \cdot \nabla v d \Omega=\int_{\Omega} f v d \Omega \quad \forall v \in \mathcal{U}
$$

where $\mathcal{U} \equiv\left\{u \in \mathcal{H}^{1}(\Omega)|u|_{\Gamma}=0\right\}$ and $\mathcal{H}^{1}(\Omega)$ is the space of all functions which are square integrable and whose first derivatives are square integrable. This problem can also be written as a minimization statement where the exact solution $u$, is the function that minimizes the total energy with respect to all candidates in $\mathcal{U}$,

$$
u=\arg \min _{\omega \in \mathcal{U}} \frac{1}{2} \int_{\Omega} \nabla \omega \cdot \nabla \omega d \Omega-\int_{\Omega} f \omega d \Omega
$$

\subsection{Output Functional}

We now express the output $s$ as a linear functional written as:

$$
s=\int_{\Omega} f^{\circ} u d \Omega
$$

where the field variable $u$ is the exact solution to the Poisson's equation in (1), and $f^{\circ}$ is some function defined over the space of polynomials $\mathbb{P}^{r}$ with degree less than or equal to $r$. Therefore we seek sharp bounds $s^{ \pm}$such that $s^{-} \leq s \leq s^{+}$.

\subsection{Continuity Relaxation}

The global domain $\Omega$ is decomposed into a mesh $\mathcal{T}_{h}$ of non-overlapping open elemental subdomains, $T$ for which $\bigcup_{T \in \mathcal{T}_{h}} \bar{T}=\bar{\Omega}$. Lagrange multipliers are introduced to enforce compatibility at the interface nodes after relaxing the continuity requirements along the faces of each subdomain. The 
boundary of each elemental subdomain is denoted by $\partial T$, with faces $\gamma$, and $\partial \mathcal{T}_{h}$, denotes all the faces in the mesh. In addition, we denote the broken space by

$$
\hat{\mathcal{U}} \equiv\left\{v \in L^{2}(\Omega),|v|_{T} \in \mathcal{H}^{1}(T), \forall T \in \mathcal{T}_{h}\right\} .
$$

Explicitly enforcing continuity along the faces of each subdomain, will lead to the equation

$$
\sum_{T \in \mathcal{T}_{h}} \int_{\partial T} \sigma_{T} \lambda \hat{\omega} d \Gamma=0 \quad \forall \lambda \in \Lambda, \forall \hat{\omega} \in \hat{\mathcal{U}}
$$

where $\sigma_{T}(x)$ depends on the arbitrary ordering of the elements and for any elements $T, T_{N} \in \mathcal{T}_{h}$

$$
\sigma_{T}(x)= \begin{cases}-1, & x \in \bar{T} \cap \bar{T}_{N}, T<T_{N}, \\ +1, & \text { otherwise. }\end{cases}
$$

The term on the left side of equation (3) also includes the Dirichlet boundaries of our problem. Note that we do not have Neumann boundary conditions in the model problem. As we shall see, equation (3) is then imposed as an additional constraint in the Lagrangian, where the $\lambda$ 's can then be computed through the gradient condition. The faces functions $\lambda$ are members of the dual trace space $\Lambda=\prod_{T \in \mathcal{T}_{h}} \mathcal{H}^{-\frac{1}{2}}(\partial T)$.

\subsection{Constrained Minimization}

We can now reformulate a variational statement of the Poisson's problem with homogeneous Dirichlet data, such that the minimization will lead to the linear output functional. Here the constraints of the Lagrangian include enforcing the continuity along the edges of the subdomain, and the equilibrium equation. To obtain both upper and lower bounds, two cases are considered which vary by the sign in front of the first term (original output) in the Lagrangian below. We write the following Lagrangian:

$$
\begin{aligned}
\mathcal{L}^{ \pm}\left(\hat{\omega}^{ \pm} ; \psi^{ \pm}, \lambda^{ \pm}\right) \equiv \mp \int_{\Omega} f^{\circ} \hat{\omega}^{ \pm} d \Omega \\
+\frac{\kappa}{2}\left\{\int_{\Omega} \nabla \hat{\omega}^{ \pm} \cdot \nabla\left(\hat{\omega}^{ \pm}-\bar{u}\right) d \Omega-\int_{\Omega} f\left(\hat{\omega}^{ \pm}-\bar{u}\right) d \Omega\right\} \\
+\int_{\Omega} f \psi^{ \pm} d \Omega-\int_{\Omega} \nabla \hat{\omega}^{ \pm} \cdot \nabla \psi^{ \pm} d \Omega \\
\quad-\sum_{T \in \mathcal{T}_{h}} \int_{\partial T} \sigma_{T} \lambda^{ \pm} \hat{\omega}^{ \pm} d \Gamma,
\end{aligned}
$$

for any $\bar{u} \in \mathcal{U}$ and any positive scaling parameter $\kappa$ which is used to tighten the bounds and to produce dimensional consistency. The output bounds are arrived at through the saddle point property of Lagrange multipliers and from the strong duality of convex minimization which leads to the inequality:

$$
\inf _{\hat{\omega} \pm \hat{\mathcal{U}}} \mathcal{L}^{ \pm}\left(\hat{\omega}^{ \pm} ; \tilde{\psi}^{ \pm}, \tilde{\lambda}^{ \pm}\right) \leq \sup _{\substack{\psi^{ \pm} \in \mathcal{U} \\ \lambda^{ \pm} \in \Lambda}} \inf _{\hat{\omega}^{ \pm} \in \hat{\mathcal{U}}} \mathcal{L}^{ \pm}\left(\hat{\omega}^{ \pm} ; \psi^{ \pm}, \lambda^{ \pm}\right)=\mp s
$$

for some candidate Lagrange multipliers $\left(\tilde{\psi}^{ \pm}, \tilde{\lambda}^{ \pm}\right) \in \mathcal{U} \times \Lambda$. The bound (5) is uncomputable in general since it requires the solution of an infinite dimensional problem. We follow the work presented 
in [24] in order to procure computable upper and lower bounds on the bound in (5). This task first requires the approximate solutions to the Lagrange multipliers $\lambda_{h}^{(k)}$, and the decoupled solutions, $u_{h}^{(k)}, \psi_{h}^{(k)}$ where the subscript $k$ denotes the restriction of the field solution to a subdomain. These approximations are then used in several subdomain calculations.

The approximation spaces we use for the finite dimensional problem are

$$
\begin{aligned}
\mathcal{U}_{h} & \equiv\left\{v \in \mathcal{U}|v|_{T} \in \mathbb{P}^{p}(T), \forall T \in \mathcal{T}_{h}\right\} \\
\Lambda_{h} & \equiv\left\{\lambda \in \Lambda|\lambda|_{\gamma} \in \mathbb{P}^{p}(\gamma), \forall \gamma \in \partial \mathcal{T}_{h}\right\}
\end{aligned}
$$

for $\mathbb{P}^{p}(T)$ being the space of polynomials on element $T$ (in three-space dimensions) with degree less than or equal to $p$, and $\mathbb{P}^{p}(\gamma)$ being the space of polynomials on element edge $\gamma$ (in two dimensions) with degree less than or equal to $p$. Moreover, the global representation of the broken space is given by

$$
\hat{\mathcal{U}}_{h} \equiv\left\{v \in \hat{\mathcal{U}}|v|_{T} \in \mathbb{P}^{p}(T), \forall T \in \mathcal{T}_{h}\right\} .
$$

The candidate Lagrange multipliers are obtained by solving the discrete form of the first variation of the Lagrangian. Upon imposing the decompositions $\lambda^{ \pm}=\frac{\kappa}{2} \lambda_{h}^{u} \pm \lambda_{h}^{\psi}, \psi^{ \pm}= \pm \psi_{h}$ and $\bar{u}=u_{h}$ the gradient condition of the Lagrangian leads to two global calculations and two equilibration equations which yield the hybrid flux approximations. These resulting set of equations are:

1. Global Approximations

(a) Primal Problem: Find $u_{h} \in \mathcal{U}_{h}$ such that

$$
\int_{\Omega} \nabla u_{h} \cdot \nabla v d \Omega=\int_{\Omega} f v d \Omega \quad \forall v \in \mathcal{U}_{h}
$$

(b) Dual Problem: Find $\psi_{h} \in \mathcal{U}_{h}$ such that

$$
\int_{\Omega} \nabla \psi_{h} \cdot \nabla v d \Omega=-\int_{\Omega} f^{\circ} v d \Omega \quad \forall v \in \mathcal{U}_{h}
$$

2. Hybrid flux calculations:

(a) Find $\lambda_{h}^{u} \in \Lambda_{h}$ such that

$$
\sum_{T \in \mathcal{T}_{h}} \int_{\partial T} \sigma_{T} \lambda_{h}^{u} \hat{v} d \Gamma-\int_{\Omega} \nabla u_{h} \cdot \nabla \hat{v} d \Omega=-\int_{\Omega} f \hat{v} d \Omega \quad \forall \hat{v} \in \hat{\mathcal{U}}_{h}
$$

(b) Find $\lambda_{h}^{\psi} \in \Lambda_{h}$ such that

$$
\sum_{T \in \mathcal{T}_{h}} \int_{\partial T} \sigma_{T} \lambda_{h}^{\psi} \hat{v} d \Gamma+\int_{\Omega} \nabla \psi_{h} \cdot \nabla \hat{v} d \Omega=-\int_{\Omega} f^{\circ} \hat{v} d \Omega \quad \forall \hat{v} \in \hat{\mathcal{U}}_{h}
$$

The hybrid flux approximations $\lambda_{h}^{u}$ and $\lambda_{h}^{\psi}$ in (8)-(9) may or may not require a priori knowledge of $u_{h}^{(k)}$ and $\psi_{h}^{(k)}$ depending on the approach chosen. The FETI based approach [17] does not require the knowledge of the $u_{h}^{(k)}$ and $\psi_{h}^{(k)}$ while the more classical approach proposed by Ladeveze and Leguillon [11] does. One may also use flux-free approaches [22]. In this work we use the FETI approach for the Poisson problem and the more efficient directly equilibrated approach described in [8] for the Stokes problem. 


\subsection{Local Dual Subproblems}

In this section we will only present the main idea and results of [24] which relates to the construction of the bounds on the exact outputs. Upon restriction to a single elemental subproblem $T \in \mathcal{T}_{h}$ the local Lagrangian is written as:

$$
\begin{aligned}
\mathcal{L}_{T}^{ \pm}\left(\omega^{ \pm} ; \pm \tilde{\psi}, \frac{\kappa}{2} \tilde{\lambda}^{u} \pm \tilde{\lambda}^{\psi}\right) \equiv \frac{\kappa}{2} \int_{T} \nabla \omega^{ \pm} \cdot \nabla \omega^{ \pm} d \Omega \\
-\frac{\kappa}{2}\left\{\int_{T}(f-\Delta \bar{u}) \omega^{ \pm} d \Omega+\int_{\partial T}\left(\sigma_{T} \tilde{\lambda}^{u}+\nabla \bar{u} \cdot \mathbf{n}\right) \omega^{ \pm} d \Gamma-\int_{T} f \bar{u} d \Omega\right\} \\
\quad \mp\left\{\int_{T}\left(f^{\circ}-\Delta \tilde{\psi}\right) \omega^{ \pm} d \Omega+\int_{\partial T}\left(\sigma_{T} \tilde{\lambda}^{\psi}+\nabla \tilde{\psi} \cdot \mathbf{n}\right) \omega^{ \pm} d \Gamma-\int_{T} f \tilde{\psi} d \Omega\right\}
\end{aligned}
$$

where Green's identity: $-\int_{T} \nabla u \cdot \nabla \omega d \Omega=\int_{T} \Delta u \omega d \Omega-\int_{\partial T} \nabla u \cdot \mathbf{n} \omega d \Gamma$ is used to ensure that no term other than the dissipative term $\frac{\kappa}{2} \int_{T} \nabla u \cdot \nabla \omega d \Omega$ involves derivatives of $\omega^{ \pm} . \bar{u}$ is any element belonging to the space $\mathcal{U}$, and $\tilde{\lambda}^{u}, \tilde{\lambda}^{\psi}$, and $\tilde{\psi}$ are candidate Lagrange multipliers. The Lagrangian can now be written as

$$
\mathcal{L}_{T}^{ \pm}\left(\omega^{ \pm} ; \pm \tilde{\psi}, \frac{\kappa}{2} \tilde{\lambda}^{u} \pm \tilde{\lambda}^{\psi}\right)=\frac{\kappa}{2} \int_{T} f \bar{u} d \Omega \pm \int_{T} f \tilde{\psi} d \Omega+J_{T}^{ \pm}\left(\omega^{ \pm}\right)
$$

where

$$
J_{T}^{ \pm}\left(\omega^{ \pm}\right) \equiv \frac{\kappa}{2} \int_{T} \nabla \omega^{ \pm} \cdot \nabla \omega^{ \pm} d \Omega-\int_{T} f^{ \pm} \omega^{ \pm} d \Omega-\int_{\partial T} g^{ \pm} \omega^{ \pm} d \Gamma
$$

for

$$
\begin{aligned}
f^{ \pm} & \equiv \frac{\kappa}{2}\{f-\Delta \bar{u}\} \pm\left\{f^{\circ}-\Delta \tilde{\psi}\right\} \\
g^{ \pm} & \equiv \frac{\kappa}{2}\left\{\sigma_{T} \tilde{\lambda}^{u}+\nabla \bar{u} \cdot \mathbf{n}\right\} \pm\left\{\sigma_{T} \tilde{\lambda}^{\psi}+\nabla \tilde{\psi} \cdot \mathbf{n}\right\} .
\end{aligned}
$$

For a positive functional

$$
J_{T}^{c}(\mathbf{q}) \equiv \frac{1}{2} \int_{T} \mathbf{q} \cdot \mathbf{q} d \Omega
$$

where $\mathbf{q} \in \mathcal{H}($ div $; T)$ it is shown $[24]$ that

$$
J_{T}^{ \pm}\left(\omega^{ \pm}\right) \geq-\frac{1}{\kappa} J_{T}^{c}\left(\mathbf{q}^{ \pm}\right),
$$

provided that $\mathbf{q}^{ \pm}$is chosen from the finite dimensional set

$$
\begin{aligned}
Q^{ \pm}(T) \equiv\{\mathbf{q} \in \mathcal{H}(\operatorname{div} ; T) \mid & \int_{T} \nabla \cdot \mathbf{q} v d \Omega-\int_{\partial T} \mathbf{q} \cdot \mathbf{n} v d \Gamma \\
& \left.=-\int_{T} f^{ \pm} v d \Omega-\int_{\partial T} g^{ \pm} v d \Gamma, \forall v \in \mathcal{H}^{1}(T)\right\} .
\end{aligned}
$$

It is now apparent from (5) and (10) for the particular choice $\bar{u}=u_{h}$ and $\tilde{\psi}=\psi_{h}$ that the local contribution of the output bounds are:

$$
\mp s_{T}^{ \pm}=\frac{\kappa}{2} \int_{T} f u_{h} d \Omega \pm \int_{T} f \psi_{h} d \Omega+\sup _{\mathbf{q}^{ \pm} \in Q^{ \pm}(T)}-\frac{1}{\kappa} J_{T}^{c}\left(\mathbf{q}^{ \pm}\right) .
$$


We recall that the data $u_{h}, \psi_{h}, \lambda_{h}^{u}$ and $\lambda_{h}^{\psi}$ in the right hand side of the dual feasibility constraint (13) are polynomial approximations of order $p$, based on the local finite element basis functions. Therefore a suitable approximation space for the dual feasibility constraint can be chosen to be

$$
Q^{ \pm, q} \equiv Q^{ \pm} \cap\left(\mathbb{P}^{q}(T)\right)^{3},
$$

where the choice of the polynomial approximation $q$ is summarized below and explained in Lemma 3.3 of [24]. The decomposition $\mathbf{q}_{h}^{ \pm}=\kappa \nabla \bar{u}+\frac{\kappa}{2} \mathbf{q}_{h}^{u} \pm \mathbf{q}_{h}^{\psi}$, in the minimization (12) leads to the two independent subproblem calculations:

$$
\begin{aligned}
& \mathbf{q}_{h}^{u}=\arg \inf _{\mathbf{q}_{h} \in Q_{h}^{u}(T)} J^{c}\left(\mathbf{q}_{h}\right), \\
& \mathbf{q}_{h}^{\psi}=\arg \inf _{\mathbf{q}_{h} \in Q_{h}^{\psi}(T)} J^{c}\left(\mathbf{q}_{h}\right),
\end{aligned}
$$

for the dual feasibility approximation sets:

$$
\begin{aligned}
\mathcal{Q}_{h}^{u}(T) \equiv\left\{\mathbf{q} \in\left(\mathbb{P}^{q}(T)\right)^{d} \mid \int_{T} \nabla \cdot \mathbf{q} v d \Omega\right. & -\int_{\partial T} \mathbf{q} \cdot \mathbf{n} v d \Omega= \\
& \left.-\int_{T}\left(f v-\nabla u_{h} \cdot \nabla v\right) d \Omega-\int_{\partial T} \sigma_{T} \lambda_{h}^{u} v d \Gamma\right\} \\
\mathcal{Q}_{h}^{\psi}(T) \equiv\left\{\mathbf{q} \in\left(\mathbb{P}^{q}(T)\right)^{d} \mid \int_{T} \nabla \cdot \mathbf{q} v d \Omega\right. & -\int_{\partial T} \mathbf{q} \cdot \mathbf{n} v d \Omega= \\
& \left.-\int_{T}\left(f^{\circ} v+\nabla \psi_{h} \cdot \nabla v\right) d \Omega-\int_{\partial T} \sigma_{T} \lambda_{h}^{\psi} v d \Gamma\right\} .
\end{aligned}
$$

for the particular choice of $\bar{u}, \tilde{p s i}, \tilde{\lambda}^{u}$ and $\tilde{\lambda}^{\psi}$ taking respectively the values $u_{h}, p s i_{h}, \lambda_{h}^{u}$ and $\lambda_{h}^{\psi}$. The existence of a solution is guaranteed by Lemma 3.3 of [24] for forcing functions $\left.f\right|_{T}$, and output functions $\left.f^{\circ}\right|_{T}$ a member of $\mathbb{P}^{r}(T)$, with the candidate Lagrange multipliers $\lambda_{h}^{u}$ and $\lambda_{h}^{\psi}$ satisfying the equilibration equations (8) and (9) respectively, and $\mathbf{q}_{h}^{u}$ a member of $Q_{h}^{u}(T)$, and $\mathbf{q}_{h}^{\psi}$ a member of $Q_{h}^{\psi}(T)$, for $q \geq p, q>r$. Moreover, it can be easily verified (see Lemma 2.5 of [24] for details) that for test functions $v \in \hat{\mathcal{U}}_{h}$, the equations (8),(17), and (9),(18) both imply the orthogonality

$$
\sum_{T \in \mathcal{T}_{h}} \int_{T} \mathbf{q} \cdot \nabla v d \Omega=0 \quad \forall T,\left.\quad \mathbf{q}_{h}^{u}\right|_{T} \in Q_{h}^{u}(T),\left.\quad \mathbf{q}_{h}^{\psi}\right|_{T} \in Q_{h}^{\psi}(T)
$$

The right hand side of (17) and (18) are localized residuals forms, and are non-zero only for the appropriate choice of polynomial approximations of both the primal and dual variables. These set of polynomial fields then allows for the certification of the bounds.

\subsection{Bounds}

With the splitting described above, summing the local contributions in (14), leads to

$$
s_{h}^{ \pm}=-\int_{\Omega} f \psi_{h}+\frac{1}{2} \int_{\Omega} \hat{\mathbf{q}}_{h}^{u} \cdot \hat{\mathbf{q}}_{h}^{\psi} d \Omega \pm \frac{\kappa}{4} J^{c}\left(\hat{\mathbf{q}}_{h}^{u}\right) \pm \frac{1}{\kappa} J^{c}\left(\hat{\mathbf{q}}_{h}^{\psi}\right)
$$


where we have invoked the orthogonality condition (19). Letting

$$
\bar{s}_{h}=\frac{1}{2} \int_{\Omega} \hat{\mathbf{q}}_{h}^{u} \cdot \hat{\mathbf{q}}_{h}^{\psi} d \Omega-\int_{\Omega} f \psi_{h} d \Omega, z_{h}^{u}=\frac{1}{4} J^{c}\left(\hat{\mathbf{q}}_{h}^{u}\right), z_{h}^{\psi}=J^{c}\left(\hat{\mathbf{q}}_{h}^{\psi}\right)
$$

we have for the optimal value of $\kappa=\sqrt{\frac{z_{h}^{\psi}}{z_{h}^{u}}}$ the bounds:

$$
s_{h}^{ \pm}=\bar{s}_{h} \pm 2 \sqrt{z_{h}^{u} z_{h}^{\psi}}
$$

where $\bar{s}_{h}$ is the bound average.

\subsection{Numerical Examples}

We now illustrate the method numerically, for the three-dimensional Poisson equation where the output of interest is

$$
s=\int_{T} f^{\circ} u d \Omega
$$

using the FETI approach to obtain the hybrid fluxes and the local finite element solutions [17]. A cube is consider for two different forcing $f$ functions. The output vector $f^{\circ}=1.0$ for all cases. Results are obtained on a tetrahedral mesh and are reported in Table (1). The effectivity is defined as $\Theta_{h}^{-}=\frac{\left|s-s_{h}^{-}\right|}{\left|s-s_{h}\right|}$ and $\Theta_{h}^{+}=\frac{\left|s-s_{h}^{+}\right|}{\left|s-s_{h}\right|}$. Moreover, for a constant forcing term, we have obtained results for two different approximations in which we consider a linear approximation for the finite element solutions where $u^{(k)}, \psi^{(k)}, \lambda^{(k)} \in \mathbb{P}^{1}$ and $\mathbf{q}, v \in \mathbb{P}^{2}$, and a higher-order approximation where $u^{(k)}, \psi^{(k)}, \lambda^{(k)} \in \mathbb{P}^{2}$ and $\mathbf{q}, v \in \mathbb{P}^{3}$.

\subsubsection{Constructed Exact Solution}

In this case we construct exact solution as a test for validation of the numerical results. We assume periodic solution of the form

$$
u(x, y, z)=\sin (\pi x) \sin (\pi y) \sin (\pi z)
$$

satisfying the homogeneous boundary conditions. This leads to the forcing function

$$
f(x, y, z)=3 \pi^{2} \sin (\pi x) \sin (\pi y) \sin (\pi z)
$$

which we have used in order to achieve bounds to the exact output, $s=\frac{8}{\pi^{3}}$. For linear finite element approximation where $p=1, q=2$ both the finite element approximation, and the output bounds asymptotically approach the expected convergence rate of $\mathrm{O}\left(h^{2}\right)$ as seen in Figure (2).

\subsubsection{Uniformly Forced Domain}

In the second case we take $f=-2.0$ and compare our solution to the exact solution:

$$
\begin{aligned}
s=-\frac{8}{\pi^{7}} \sum_{n=1}^{\infty} \sum_{m=1}^{\infty} & \frac{\left[1-(-1)^{n}\right]^{2}\left[1-(-1)^{m}\right]^{2}}{n^{2} m^{2}\left(n^{2}+m^{2}\right)^{3 / 2}} \times \\
& \left\{\frac{4 e^{-\pi \sqrt{n^{2}+m^{2}}}}{1-e^{-2 \pi \sqrt{n^{2}+m^{2}}}}-\frac{2\left(1+e^{-2 \pi \sqrt{n^{2}+m^{2}}}\right)}{1-e^{-2 \pi \sqrt{n^{2}+m^{2}}}}+\pi \sqrt{n^{2}+m^{2}}\right\} \approx-0.0403405
\end{aligned}
$$




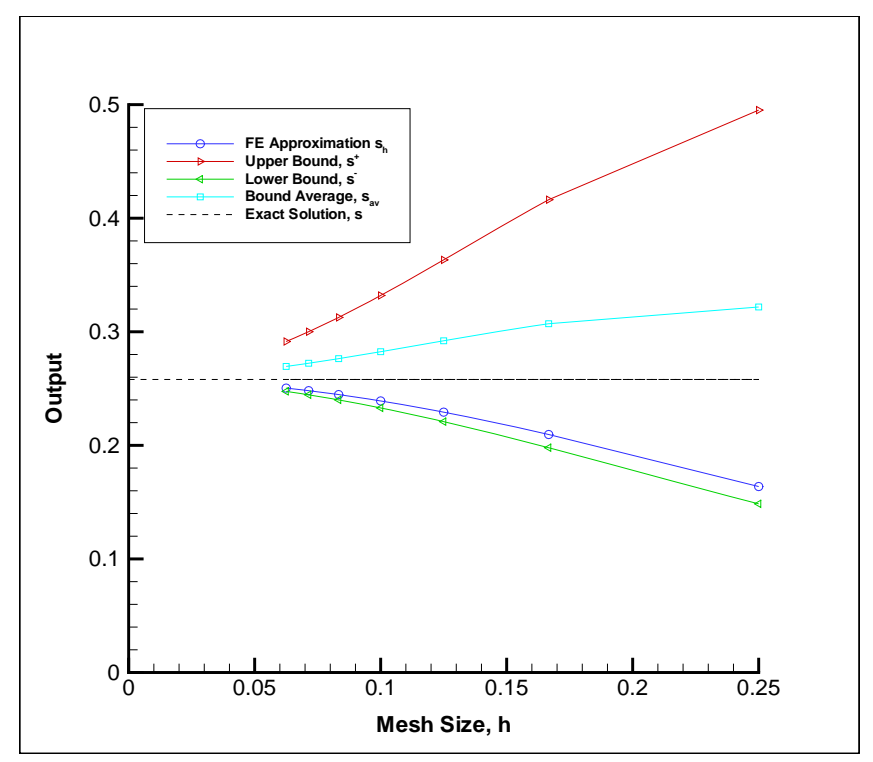

(a)

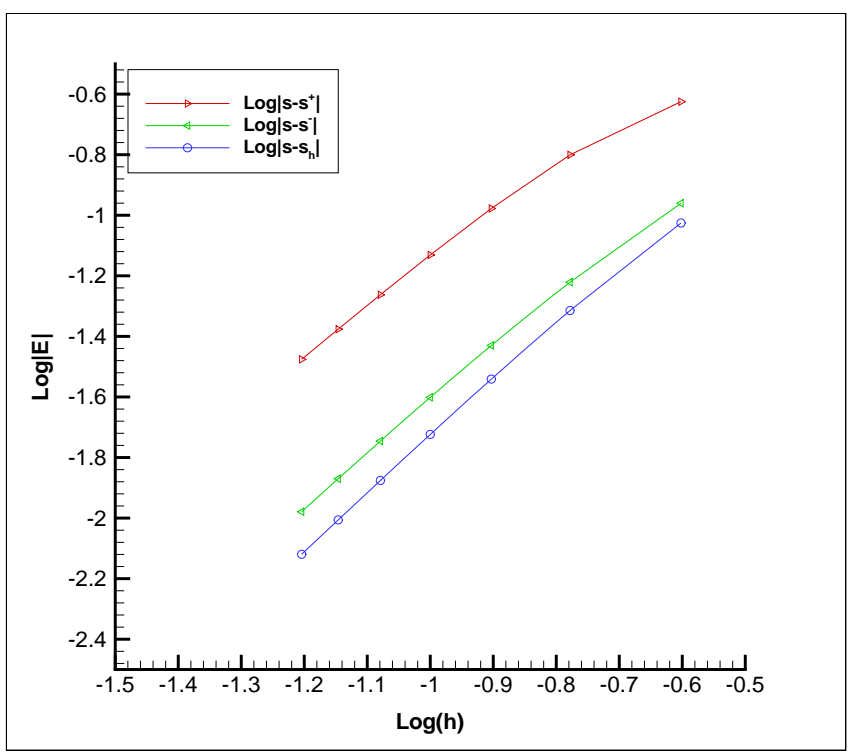

(b)

Figure 2: Bounds for the constructed solution output (a) upper and lower bounds (b) convergence of the bounds.

Note that for the optimal parameter $\kappa$, choosing a constant forcing will cause the finite element solution to be equal to either the upper bound or the lower bound depending on the sign of the forcing term. In this case, the upper bound is equal to the finite element approximation. For $p=1, q=2$, Figure (3) shows again plots of the bounds and an asymptotic convergence rate of $\mathrm{O}\left(h^{2}\right)$. However we compare this case with Figure (4) where higher order polynomial approximation, $p=2, q=3$ are used. The asymptotic convergence rate for both the output bounds and the finite element approximation are of $\mathrm{O}\left(h^{4}\right)$.

\begin{tabular}{|c|c|c|c|c|c|c|c|c|c|}
\hline \multicolumn{5}{|c|}{$\begin{array}{c}f=3 \pi^{2} \sin (\pi x) \sin (\pi y) \sin (\pi z) \\
s=8 / \pi^{3} \approx 0.2580122\end{array}$} & \multicolumn{5}{|c|}{$\begin{array}{c}f=-2.0 \\
s \approx-0.0403405\end{array}$} \\
\hline $\mathbf{h}$ & $\mathbf{s}_{\mathrm{h}}$ & $\mathrm{s}_{\mathrm{h}}^{+}$ & $\theta_{h}^{-}$ & $\theta_{h}^{+}$ & h & $\mathrm{s}_{\mathrm{h}}^{-}$ & $\mathbf{s}_{\mathrm{h}}^{+}$ & $\theta_{h}^{-}$ & $\theta_{h}^{+}$ \\
\hline $1 / 2$ & 0.038018 & 0.380210 & 1.0864 & 0.6035 & $1 / 2$ & -0.121818 & -0.009375 & 2.6312 & 1.0 \\
\hline $1 / 4$ & 0.148498 & 0.495173 & 1.1617 & 2.5157 & $1 / 4$ & -0.092205 & -0.026951 & 3.8735 & 1.0 \\
\hline $1 / 6$ & 0.197912 & 0.416287 & 1.2402 & 3.2661 & $1 / 6$ & -0.071412 & -0.033440 & 4.5028 & 1.0 \\
\hline $1 / 8$ & 0.220851 & 0.363288 & 1.2914 & 3.6585 & $1 / 8$ & -0.060518 & -0.036211 & 4.8862 & 1.0 \\
\hline $1 / 10$ & 0.232964 & 0.331964 & 1.3256 & 3.9136 & $1 / 10$ & -0.054446 & -0.037610 & 5.1659 & 1.0 \\
\hline $1 / 12$ & 0.240049 & 0.312650 & 1.3496 & 4.1049 & $1 / 12$ & -0.050774 & -0.038408 & 5.3990 & 1.0 \\
\hline $1 / 14$ & 0.244523 & 0.300063 & 1.3676 & 4.2633 & $1 / 14$ & -0.048397 & -0.038902 & 5.6006 & 1.0 \\
\hline $1 / 16$ & 0.247519 & 0.291445 & 1.3817 & 4.4024 & $1 / 16$ & -0.046772 & -0.039229 & 5.7863 & 1.0 \\
\hline
\end{tabular}

Table 1: Tabulated bounds results obtained for the tetrahdral elemental subdomains with $p=1, q=2$ 


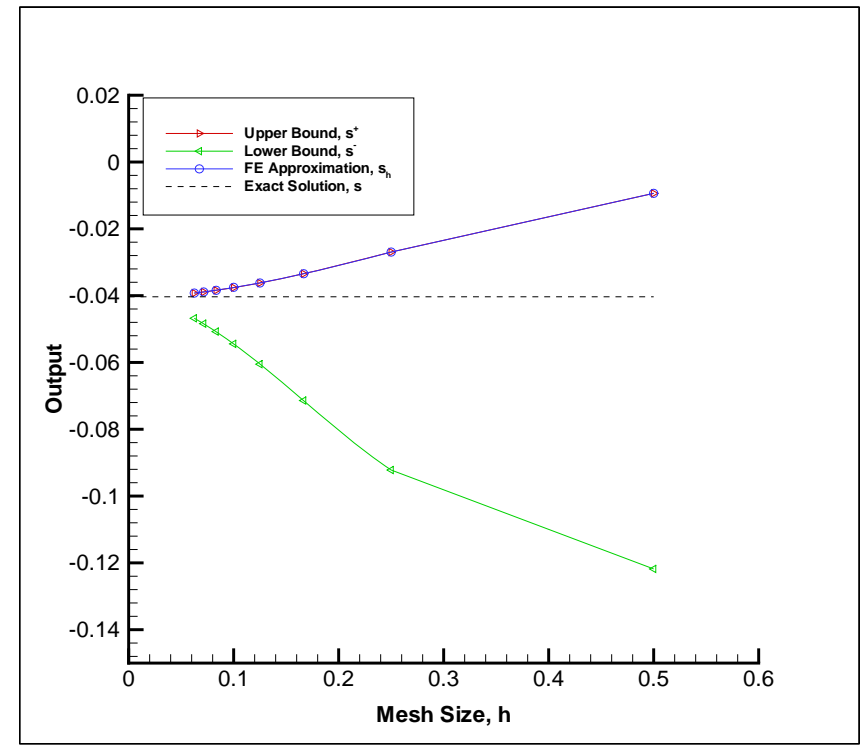

(a)

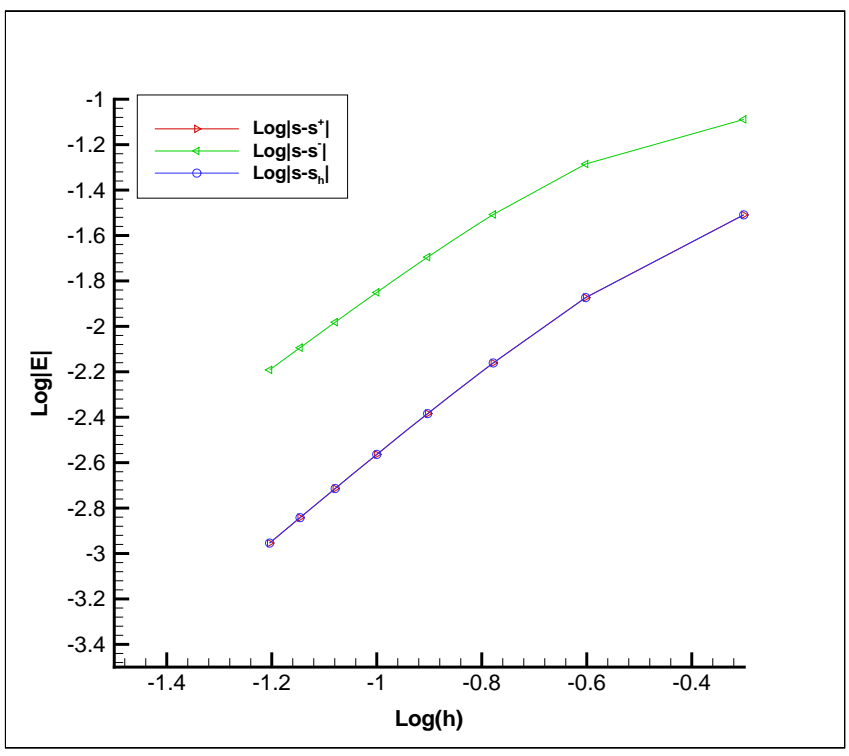

(b)

Figure 3: Bounds for the constant forcing problem $(f=-2.0)$ with $p=1, q=2(\mathbf{a})$ upper and lower bounds (b) convergence of the bounds. 


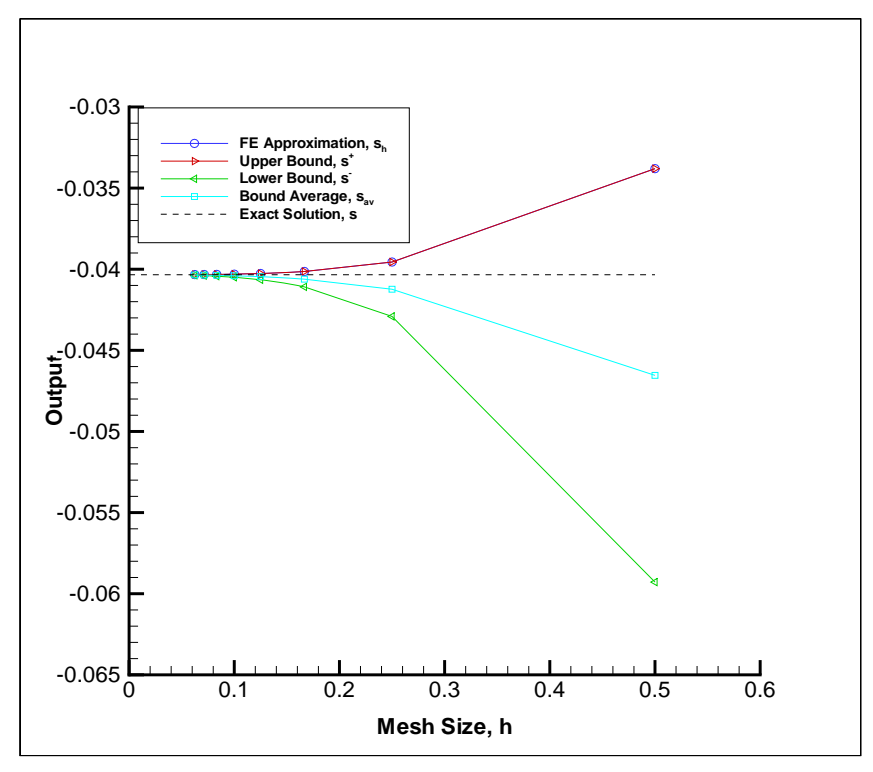

(a)

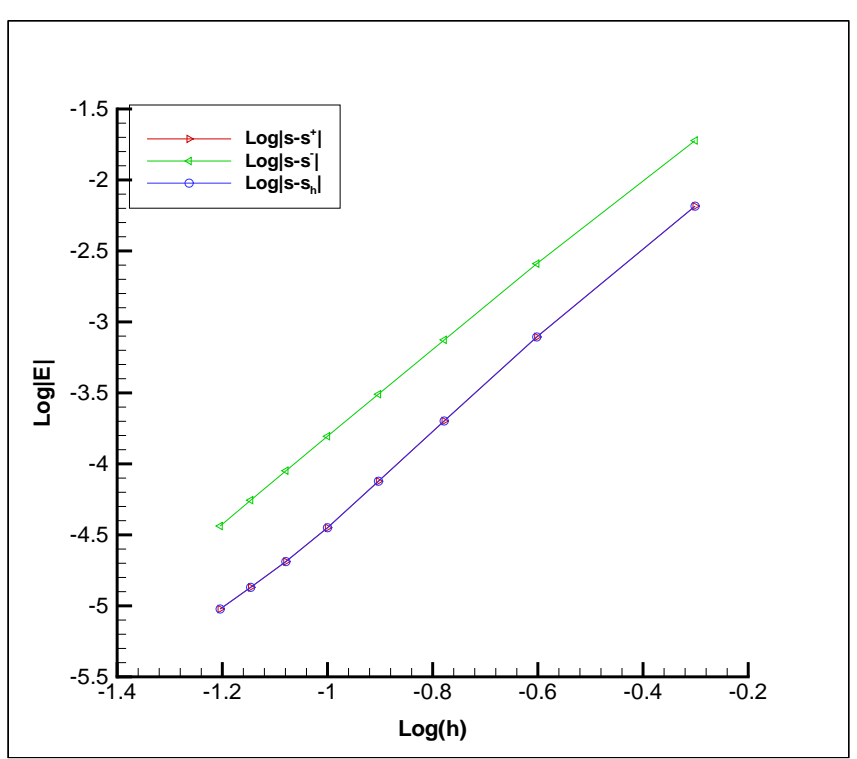

(b)

Figure 4: Bounds for the constant forcing problem $(f=-2.0)$ with $p=2, q=3$ (a) upper and lower bounds (b) convergence of the bounds.

\section{Exact Bounds Extension to Outputs of the Incompressible Stokes Problem}

In this section, we extend the previous technique to bound outputs of the incompressible Stokes problem.

\subsection{Model Problem}

Figure 5 shows the geometry of the benchmark problem which is a steady, incompressible (density $\rho=$ constant), creeping flow driven by the forcing term in an endless square channel with an array of rectangular obstacles in the center [5]. The flow is assumed to be a Newtonian fluid with a constant dynamic viscosity, $\mu$. The geometry of the domain is represented by $\Omega$ and $\left(x_{1}, x_{2}, x_{3}\right)$ denotes the coordinate system, with corresponding unit vectors $\vec{x}_{1}, \vec{x}_{2}, \vec{x}_{3}$. The driving force is a pressure gradient $\frac{\Delta P}{L}$ in the $\vec{x}_{3}$ direction, where $L$ is the scaling length of the channel section, and $\Delta P$ is the pressure difference between the two reference points with the distance of $L$ in the $\vec{x}_{3}$ direction. The fluid velocity and pressure perturbations are periodic in the $\vec{x}_{3}$ direction. Let the fluid velocity be $\mathbf{u}=\left(u_{1}, u_{2}, u_{3}\right)$ with $u_{i}$ being the corresponding component in the $\vec{x}_{i}$ direction and $p$ be the pressure fluctuation field divided by the viscosity $\mu$. The governing equations for the incompressible Stokes flow can be written in indicial notation as:

$$
\begin{aligned}
-\frac{\partial^{2} u_{i}}{\partial x_{j} \partial x_{j}}+\frac{\partial p}{\partial x_{i}} & =f_{i} \text { in } \Omega, \quad i=1,2,3 \\
\frac{\partial u_{i}}{\partial x_{i}} & =0 \text { in } \Omega,
\end{aligned}
$$


with the boundary conditions:

$$
\begin{aligned}
\left.u_{i}\right|_{\Gamma_{1}} & =\left.u_{i}\right|_{\Gamma_{2}}, \\
u_{i} & =0, \text { on the other boundaries, }
\end{aligned}
$$

where $f_{i}=\frac{\Delta P}{\mu L}$ is a prescribed forcing term in the $\vec{x}_{i}$ direction. For this benchmark problem, the following values are selected: $f_{1}=f_{2}=0$ and $f_{3}=1$. For simplicity, $\mu=1$ is selected for the numerical experiments. Thus $\Omega$ is a bounded cubic domain $] 0,1[\times] 0,1[\times] 0,1[$ with a rectangular obstacle $] 0,1[\times] \frac{1}{3}, \frac{2}{3}[\times] \frac{1}{3}, \frac{2}{3}[$ inside. $\Omega=] 0,1[\times] 0,1[\times] 0,1[-] 0,1[\times] \frac{1}{3}, \frac{2}{3}[\times] \frac{1}{3}, \frac{2}{3}[$. The periodic boundaries are $\left.\Gamma_{1}=\right] 0,1[\times] 0,1\left[\right.$ at $x_{3}=1$ and $\left.\Gamma_{2}=\right] 0,1[\times] 0,1\left[\right.$ at $x_{3}=0$.

To ensure a unique solution for the pressure, an additional requirement is that the integral of the pressure perturbation over the domain is zero:

$$
\int_{\Omega} p d V=0
$$

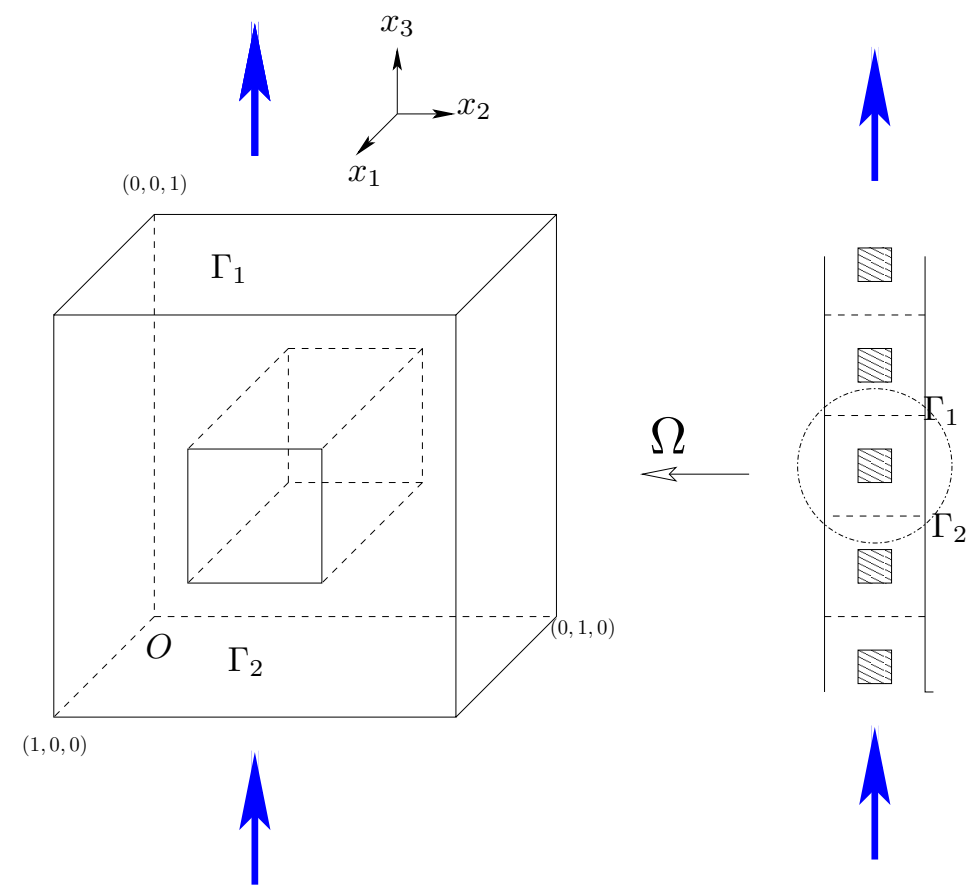

Figure 5: Geometry for the Stokes flow problem. Periodic boundary conditions are applied on $\Gamma_{1}$ and $\Gamma_{2}$ while homogeneous boundary conditions are enforced on $\partial \Omega\left(\Gamma_{1} \cup \Gamma_{2}\right)$

The corresponding variational forms follow as: Given $\left(f_{1}, f_{2}, f_{3}\right) \in\left(\mathcal{H}^{-1}(\Omega)\right)^{3}$, find $\left(u_{1}, u_{2}, u_{3}, p\right) \in Y$ such that:

$$
\begin{aligned}
\int_{\Omega} \frac{\partial \omega_{i}}{\partial x_{j}} \frac{\partial u_{i}}{\partial x_{j}} d V-\int_{\Omega} p \frac{\partial \omega_{i}}{\partial x_{i}} d V=\int_{\Omega} f_{i} \omega_{i} d V, \quad \forall\left(\omega_{1}, \omega_{2}, \omega_{3}\right) \in X \\
-\int_{\Omega} r \frac{\partial u_{i}}{\partial x_{i}} d V=0, \quad \forall r \in \mathcal{Q},
\end{aligned}
$$


where $d V$ is a differential volume element. $X, \mathcal{Q}$ and $Y$ are the function spaces defined as:

$$
\begin{aligned}
X & =\mathcal{H}_{0}^{1}(\Omega) \times \mathcal{H}_{0}^{1}(\Omega) \times \mathcal{H}_{0}^{1}(\Omega), \\
\mathcal{Q} & =L^{2}(\Omega), \\
Y & =X \times \mathcal{Q} .
\end{aligned}
$$

Defining:

$$
\begin{array}{rlrl}
a(\mathbf{v}, \mathbf{w}) & =\int_{\Omega} \frac{\partial v_{i}}{\partial x_{j}} \frac{\partial \omega_{i}}{\partial x_{j}} d V, & & \forall(\mathbf{v}, \mathbf{w}) \in X \times X, \\
b(\mathbf{v}, r)=-\int_{\Omega} r \frac{\partial v_{i}}{\partial x_{i}} d V, & & \forall(\mathbf{v}, r) \in Y, \\
\ell^{N}(\mathbf{w})=\int_{\Omega} f_{i} \omega_{i} d V, & & \forall \mathbf{w} \in X .
\end{array}
$$

Then the weak formulation of the Stokes problem is:

Find $(\mathbf{u}, p) \in Y$, such that

$$
\begin{aligned}
a(\mathbf{u}, \mathbf{w})+b(\mathbf{w}, p) & =\ell^{N}(\mathbf{w}), \quad \forall \mathbf{w} \in X \\
b(\mathbf{u}, r) & =0, \quad \forall r \in \mathcal{Q} .
\end{aligned}
$$

\subsection{Output Functional}

Engineering design is based on outputs which are functionals of the field solution. In this paper, only linear functionals are addressed and bounds are calculated to these outputs.

For the Stokes equations (21), (22), the field solution is $(\mathbf{u}, p)$, or $\left(u_{1}, u_{2}, u_{3}, p\right)$ and the output is defined by $s=s(\mathbf{u}, p)=s\left(u_{1}, u_{2}, u_{3}, p\right)$, where $\ell: Y \rightarrow \Re$. For the purpose of simplicity, only the integral of the velocity in the flow direction, $\vec{x}_{3}$ direction is considered. A quantity of interest that depends on pressure can also be defined as in [20] but we do not examine this type of output in this formulation. This quantity is a normalized flowrate where the flowrate is the output values divided by the volume of the computational domain which is $(24 / 27)$. The output is therefore defined as:

$$
s(\mathbf{u})=\int_{\Omega}(\boldsymbol{\alpha} \cdot \mathbf{u}) d \Omega
$$

where $\boldsymbol{\alpha}$ is a unit vector which indicates the direction in which the displacement is evaluated is a user defined coefficient: here $\boldsymbol{\alpha}=(0,0,1)$. Thus, the linear functional of the output of interest can be expressed as

$$
\ell^{0}(\mathbf{u})=\int_{\Omega} u_{3} d \Omega
$$

\subsection{Continuity Relaxation}

\subsubsection{Domain decomposition and tetrahedral elements}

The bounds method for the Stokes equations is based on global and local calculations. Two different discretizations on the same mesh are needed; a lower order discretization and a higher order discretization. The computational domain $\Omega$ is divided into a mesh $\mathcal{T}_{h}$ of $k$ tetrahedra $T$.

The domain $\Omega$ is also decomposed into $N_{k}$ subdomains $\Omega^{(k)}$. Note that each element of the mesh $\mathcal{T}_{h}$ is not a subdomain. For the problem addressed herein, $\Omega$ is decomposed into $N_{k}=24$ subdomains, $\Omega^{(k)}$, each of which is a uniform small cube with the same dimension of $\left(\frac{1}{3} \times \frac{1}{3} \times \frac{1}{3}\right)$, as shown in Figure 6 . Therefore each subdomain $\Omega^{(k)}$ contains a small number of tetrahedral elements. Figure 7 illustrates the $\mathcal{T}_{h}$ before and after the domain decompositon. The thick lines represent subdomain borders. 

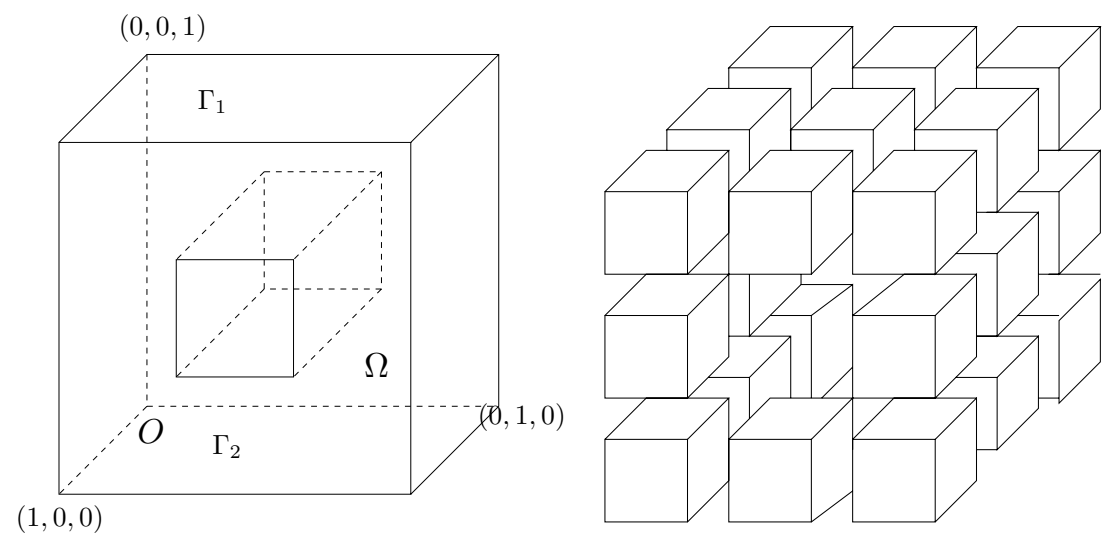

Figure 6: The domain decomposition of a unit cube for the Stokes problem
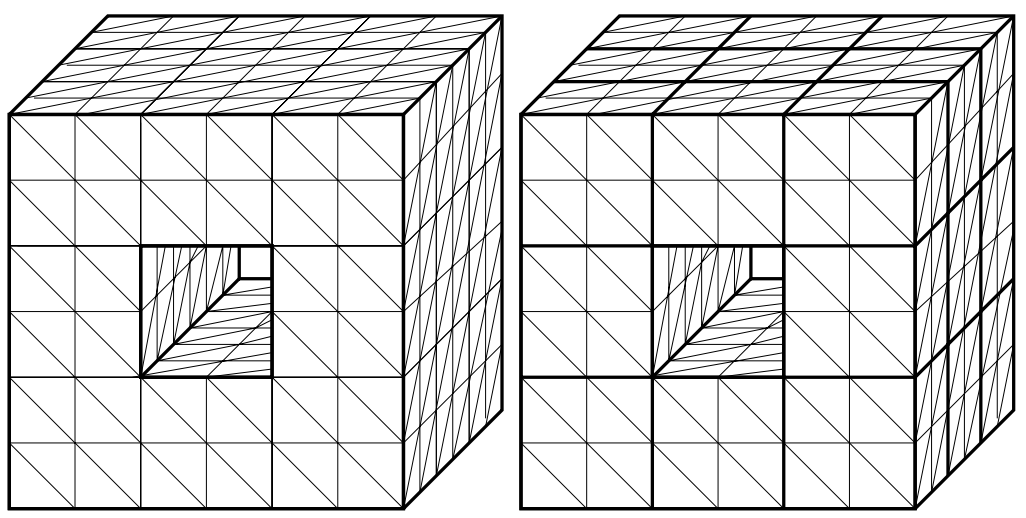

Figure 7: The mesh before domain decomposition (left) and after the domain decomposition (right)

\subsubsection{Finite element space}

Two different order polynomial spaces are used to calculate the bound. A low order polynomial space [3] and the well known Crouzeix and Raviart space [9] are defined below. The main difference is that the low order space is a piecewise linear space enhanced with "bubble" functions while the higher order space is a quadratic piecewise space also enhanced with the same "bubble" functions.

Therefore, the velocity of the lower order space is a member of $\mathbf{P}^{++}$, a linear piecewise enhanced with "bubble" functions over the element, and the pressure is a member of a discontinuous $\mathbf{P}_{1}$ space. This space satisfies the inf sup condition. Let the approximate velocity $\mathbf{u}_{h}$ be in $X_{h}$ and the pressure $p_{h}$ be in $\mathcal{Q}_{h}$, where

$$
\begin{aligned}
X_{h} & =\left\{\mathbf{u} \in X \mid u_{i \mid T} \in \mathbf{P}_{1}(T) \oplus \mathbf{B}_{4}(T), \forall T \in \mathcal{T}_{h}\right\} \\
\mathcal{Q}_{h} & =\left\{p \in L^{2}(\Omega) \mid p_{\mid T} \in \mathbf{P}_{1}(T), \forall T \in \mathcal{T}_{h}\right\}
\end{aligned}
$$

Here, $\mathbf{B}_{4}(T)$ denotes the space of bubble functions of degree 4 on $T$. Finally, the solution space is defined as the product space $Y_{h}=X_{h} \times \mathcal{Q}_{h}$.

Regarding the Crouzier-Raviart space, the velocity is a member of $\mathbf{P}_{2}^{+}$, a quadratic piecewise space enhanced with "bubble" functions over the element, and the pressure is a member of 
a discontinuous $\mathbf{P}_{1}$ space. This space is only used for the local decoupled problems. Let the approximate velocity $\hat{\mathbf{u}}_{h}$ be in $\hat{X}_{h}$ and the pressure $p_{h}$ be in $\mathcal{Q}_{h}$, where

$$
\begin{aligned}
\hat{X}_{h} & =\left\{\mathbf{u} \in X \mid u_{i \mid T} \in \mathbf{P}_{2}(T) \oplus \mathbf{B}_{4}(T), \forall T \in \mathcal{T}_{h}\right\} \\
\mathcal{Q}_{h} & =\left\{p \in L^{2}(\Omega) \mid p_{\mid T} \in \mathbf{P}_{1}(T), \forall T \in \mathcal{T}_{h}\right\} .
\end{aligned}
$$

Finally, the solution space is defined as the product space $\hat{Y}_{h}=\hat{X}_{h} \times \mathcal{Q}_{h}$.

The discontinuous pressure is ideal for the bound method because pressure continuity must not be enforced in the Lagrangian. Note that the global calculations use the lower order element and only the local calculations use the more expensive Crouzeix-Raviart element.

\subsubsection{The discrete problem}

To calculate the discrete output of interest, $s_{h}$, we need to find $\left(\mathbf{u}_{h}, p_{h}\right) \in X_{h} \times \mathcal{Q}_{h}$ such that

$$
\begin{aligned}
a\left(\mathbf{u}_{h}, \mathbf{w}\right)+b\left(\mathbf{w}, p_{h}\right)-\ell^{N}(\mathbf{w}) & =0, \quad \forall \mathbf{w} \in X_{h}, \\
b\left(\mathbf{u}_{h}, r\right) & =0, \quad \forall r \in \mathcal{Q}_{h} ;
\end{aligned}
$$

and then to compute the output,

$$
s_{h}=\ell^{0}\left(u_{1 h}, u_{2 h}, u_{3 h}\right) .
$$

\subsubsection{Spaces for subdomain edges}

The finite element edge space $\Gamma\left(\mathcal{T}_{h}\right)$ denotes the set of open faces and edges $\gamma_{h}$ between different subdomains discretized on the mesh tetrahedra $\mathcal{T}_{h}$.

The "broken" spaces of each subdomain $\Omega^{(k)}$ are defined as:

$$
\begin{aligned}
\hat{X}_{h}^{\text {low }} & =\left\{\mathbf{v} \in H^{1}(T) \times H^{1}(T) \times H^{1}(T) \mid v_{\left.i\right|_{T}} \in \mathbf{P}_{1}(T) \oplus \mathbf{B}_{4}(T), \forall T \in \mathcal{T}_{h}\right\}, \\
\hat{X}_{h}^{\text {high }} & =\left\{\mathbf{v} \in H^{1}(T) \times H^{1}(T) \times H^{1}(T)\left|v_{i}\right|_{T} \in \mathbf{P}_{2}(T) \oplus \mathbf{B}_{4}(T), \forall T \in \mathcal{T}_{h}\right\},
\end{aligned}
$$

If $\hat{Y}_{h}^{\text {low }}=\hat{X}_{h}^{\text {low }} \times \mathcal{Q}_{h}$ and $\hat{Y}_{h}=\hat{X}_{h}^{\text {high }} \times \mathcal{Q}_{h}$, there exist $\hat{Y}_{h}^{\text {low }} \subset \hat{Y}_{h}$.

The continuity of the three velocity components is enforced using a hybrid flux vector that has three components $\mathbf{t}=\left(t_{1}, t_{2}, t_{3}\right)$. The "hybrid flux" space associated to this vector is defined as

$$
\mathbf{E}_{h}^{\text {low }}=\left\{\mathbf{t} \in L^{2}\left(\Gamma\left(\mathcal{T}_{h}\right)\right) \times L^{2}\left(\Gamma\left(\mathcal{T}_{h}\right)\right) \times L^{2}\left(\Gamma\left(\mathcal{T}_{h}\right)\right) \mid t_{i \mid \gamma_{h}} \in \mathbf{P}_{1}\left(\gamma_{h}\right) \oplus \mathbf{B}_{4}\left(\gamma_{h}\right), \forall \gamma_{h} \in \Gamma\left(\mathcal{T}_{h}\right)\right\} .
$$

Similarly, for the higher order discretization, it is defined as

$$
\mathbf{E}_{h}=\left\{\mathbf{t} \in L^{2}\left(\Gamma\left(\mathcal{T}_{h}\right)\right) \times L^{2}\left(\Gamma\left(\mathcal{T}_{h}\right)\right) \times L^{2}\left(\Gamma\left(\mathcal{T}_{h}\right)\right) \mid t_{i \mid \gamma_{h}} \in \mathbf{P}_{2}\left(\gamma_{h}\right) \oplus \mathbf{B}_{4}\left(\gamma_{h}\right), \forall \gamma_{h} \in \Gamma\left(\mathcal{T}_{h}\right)\right\} .
$$

where $i=1,2,3$. It follows that $\mathbf{E}_{h}^{\text {low }} \subset \mathbf{E}_{h} \subset \mathcal{H}^{-1 / 2}\left(\Gamma\left(\mathcal{T}_{h}\right)\right)$; the functions in these spaces can, of course, be discontinuous.

The jump bilinear form $H(\cdot, \cdot)$ is considered here: $\hat{X}_{h} \times \mathbf{E}_{h} \rightarrow \Re$,

$$
H(\mathbf{v}, \mathbf{t})=\sum_{\gamma_{h} \in \Gamma\left(\mathcal{T}_{h}\right)} \int_{\gamma_{h}} \mathcal{J}_{\gamma_{h}} v_{i} t_{\left.i\right|_{\gamma_{h}}} d s
$$

where $i=1,2,3 . \mathcal{J}_{\gamma_{h}} v_{i}$ is the jump in $v_{i}$ across $\gamma_{h}$ when $\gamma_{h}$ is an interior face, and the trace of $v_{i}$ on $\gamma_{h}$ when $\gamma_{h}$ is on the boundary $\partial \Omega$. Note that $t_{i}$ is defined only over the faces of the subdomain and may, of course, be discontinuous; $t_{i}$ may also be defined as the flux associated with a function in $\mathbf{E}_{h}$.

The hybrid flux can be used to enforce continuity on functions in $\hat{X}_{h}^{\text {low }}$ and $\hat{X}_{h}$; in particular,

$$
\begin{aligned}
X_{h}^{\text {low }} & =\left\{\mathbf{v} \in \hat{X}_{h}^{\text {low }} \mid H(\mathbf{v}, \mathbf{t})=0, \forall \mathbf{t} \in \mathbf{E}_{h}^{\text {low }}\right\}, \\
X_{h} & =\left\{\mathbf{v} \in \hat{X}_{h} \mid H(\mathbf{v}, \mathbf{t})=0, \forall \mathbf{t} \in \mathbf{E}_{h}\right\} .
\end{aligned}
$$




\subsection{Constrained Minimization}

Following the same procedure as in Section 2.4, we construct a Lagrangian where the constraints include the Stokes finite element equilibrium equations and the inter-subdomain continuity requirements such as $\mathcal{L}: \hat{X} \times \mathcal{Q} \times X \times \mathcal{Q} \times \mathbf{E} \rightarrow \Re$,

$$
\begin{aligned}
\mathcal{L}^{ \pm}(\mathbf{v}, \beta, \mathbf{w}, r, \mu)= & \kappa\left\{a(\mathbf{v}, \mathbf{v})+2 b(\mathbf{v}, \beta)-\ell^{N}(\mathbf{v})+a(\overline{\mathbf{u}}, \mathbf{v})+b(\mathbf{v}, \bar{p})+b(\overline{\mathbf{u}}, \beta)\right\} \pm \ell^{0}(\overline{\mathbf{u}}+\mathbf{v}) \\
& +\left\{a(\overline{\mathbf{u}}+\mathbf{v}, \mathbf{w})-\ell^{N}(\mathbf{w})+b(\mathbf{w}, \bar{p}+\beta)+b(\overline{\mathbf{u}}+\mathbf{v}, r)\right\} \\
& +H(\mathbf{v}, \mu)
\end{aligned}
$$

where the first line represents the quadratic "energy" reformation of the desired output, the second line represents the original Stokes problem constrained by the Lagrange multipliers $\mathbf{w}$ and $r$, and the third line represents the hybrid flux constraints that "glue" the subdomains together. Details of the construction of this Lagrangian is given in Appendix A. Note that $\hat{X}$ is defined as:

$$
\hat{X} \equiv\left\{v\left|v \in L^{2}(\Omega), v\right|_{T} \in \mathcal{H}^{1}(T), \forall T \in \mathcal{T}_{h}\right\} .
$$

As for the Poisson problem, for the case $\overline{\mathbf{u}}=\mathbf{u}_{h} \in \hat{X}_{h}, \bar{p}=p_{h} \in \mathcal{Q}_{h}$, we introduce candidate Lagrange multipliers obtained from the finite element solution $\mathbf{w}=\psi_{h}^{ \pm}= \pm \psi_{h} \in X_{h}, r=\theta_{h}{ }^{ \pm}=$ $\pm \theta_{h} \in \mathcal{Q}_{h}$, and $\mu=\mathbf{t}_{h}^{ \pm}=\kappa \mathbf{t}_{h}^{p r} \pm \mathbf{t}_{h}^{d u} \in \mathbf{E}_{h}$. The calculation of these Lagrange multipliers is detailed in Appendix B. The Lagrangian can now be written as

$$
\begin{aligned}
\mathcal{L}^{ \pm}(\mathbf{v}, \beta, \mathbf{w}, r, \mu) \\
=\mathcal{L}^{ \pm}\left(\mathbf{v}, \beta, \pm \psi_{h}, \pm \theta_{h}, \kappa \mathbf{t}_{h}^{p r} \pm \mathbf{t}_{h}^{d u}\right) \\
=\kappa a(\mathbf{v}, \mathbf{v})+2 \kappa b(\mathbf{v}, \beta) \\
\quad+\kappa\left\{a\left(\mathbf{u}_{h}, \mathbf{v}\right)+b\left(\mathbf{v}, p_{h}\right)-\ell^{N}(\mathbf{v})+H\left(\mathbf{v}, \mathbf{t}_{h}^{p r}\right)+b\left(\mathbf{u}_{h}, \beta\right)\right\} \\
\quad \pm\left\{a\left(\mathbf{v}, \psi_{h}\right)+b\left(\mathbf{v}, \theta_{h}\right)+\ell^{0}(\mathbf{v})+H\left(\mathbf{v}, \mathbf{t}_{h}^{d u}\right)+b\left(\psi_{h}, \beta\right)\right\} \\
\pm\left\{\ell^{0}\left(\mathbf{u}_{h}\right)+a\left(\mathbf{u}_{h}, \psi_{h}\right)+b\left(\psi_{h}, p_{h}\right)-\ell^{N}\left(\psi_{h}\right)+b\left(\mathbf{u}_{h}, \theta_{h}\right)\right\}
\end{aligned}
$$

From the Lagrangian (53), we can obtain the lower bound $s^{-}=\eta^{+}$and upper bound $s^{+}=$ $-\eta^{-}$by minimizing:

$$
\eta^{ \pm} \equiv \inf _{\mathbf{v} \in \hat{X}_{h}} \kappa a(\mathbf{v}, \mathbf{v})+\mathbf{L}^{ \pm}(\mathbf{v}, \beta)+\mathbf{C}^{ \pm}
$$

where

$$
\begin{aligned}
\mathbf{L}^{ \pm}(\mathbf{v}, \beta)= & 2 \kappa b(\mathbf{v}, \beta)+\kappa\left\{a\left(\mathbf{u}_{h}, \mathbf{v}\right)+b\left(\mathbf{v}, p_{h}\right)-\ell^{N}(\mathbf{v})+H\left(\mathbf{v}, \mathbf{t}_{h}^{p r}\right)+b\left(\mathbf{u}_{h}, \beta\right)\right\} \\
& \pm\left\{a\left(\mathbf{v}, \psi_{h}\right)+b\left(\mathbf{v}, \theta_{h}\right)+\ell^{0}(\mathbf{v})+H\left(\mathbf{v}, \mathbf{t}_{h}^{d u}\right)+b\left(\psi_{h}, \beta\right)\right\} \\
\mathbf{C}^{ \pm}= & \pm\left\{\ell^{0}\left(\mathbf{u}_{h}\right)+a\left(\mathbf{u}_{h}, \psi_{h}\right)+b\left(\psi_{h}, p_{h}\right)-\ell^{N}\left(\psi_{h}\right)+b\left(\mathbf{u}_{h}, \theta_{h}\right)\right\}
\end{aligned}
$$

As in [20], we observe that when we expand our Lagrangian in $\mathbf{v}$ for any $\beta$ all the terms vanish and the remaining are positive semi-definite terms in $a($,$) which thus prove (54).$

\subsection{Local Dual Subproblems}

Due to the continuity relaxation from our finite element choices of the Lagrange multipliers, we can now calculate output bounds as sums of elemental contributions:

$$
\eta^{ \pm}=\sum_{\Omega_{k} \in \Omega} \eta_{\Omega_{k}}^{ \pm}
$$


and the equation (54) becomes:

$$
\eta_{\Omega_{k}}^{ \pm} \equiv \inf _{\mathbf{v} \in H^{1}\left(\Omega_{k}\right)} \kappa a_{\Omega_{k}}(\mathbf{v}, \mathbf{v})+\mathbf{L}_{\Omega_{k}}^{ \pm}(\mathbf{v}, \beta)+\mathbf{C}_{\Omega_{k}}^{ \pm}
$$

where

$$
\begin{aligned}
\mathbf{L}_{\Omega_{k}}^{ \pm}(\mathbf{v}, \beta)= & 2 \kappa b_{\Omega_{k}}(\mathbf{v}, \beta)+\kappa\left\{a_{\Omega_{k}}\left(\mathbf{u}_{h}, \mathbf{v}\right)+b_{\Omega_{k}}\left(\mathbf{v}, p_{h}\right)-\ell_{\Omega_{k}}^{N}(\mathbf{v})+H_{\Omega_{k}}\left(\mathbf{v}, \mathbf{t}_{h}^{p r}\right)+b_{\Omega_{k}}\left(\mathbf{u}_{h}, \beta\right)\right\} \\
& \pm\left\{a_{\Omega_{k}}\left(\mathbf{v}, \psi_{h}\right)+b_{\Omega_{k}}\left(\mathbf{v}, \theta_{h}\right)+\ell_{\Omega_{k}}^{0}(\mathbf{v})+H_{\Omega_{k}}\left(\mathbf{v}, \mathbf{t}_{h}^{d u}\right)+b_{\Omega_{k}}\left(\psi_{h}, \beta\right)\right\} \\
\mathbf{C}_{\Omega_{k}}^{ \pm}= & \pm\left\{\ell_{\Omega_{k}}^{0}\left(\mathbf{u}_{h}\right)+a_{\Omega_{k}}\left(\mathbf{u}_{h}, \psi_{h}\right)+b_{\Omega_{k}}\left(\psi_{h}, p_{h}\right)-\ell^{N}\left(\psi_{h}\right)+b_{\Omega_{k}}\left(\mathbf{u}_{h}, \theta_{h}\right)\right\}
\end{aligned}
$$

where the subscript $\Omega_{k}$ denotes restriction to a single subdomain.

As seen in Section 2.5, the exact bound method is based on the complementary energy functional that bounds a quadratic functional defined elementally which is obtained by selecting Lagrange multipliers in the Lagrangian. In this section we show how the complementary energy function bounds the Lagrangian constructed for the output of the Stokes problem. We then derive the subdomain problem needed to obtain $\mathbf{q}$, which is a tensor: $\mathbf{q}=\left(\mathbf{q}_{1}, \mathbf{q}_{2}, \mathbf{q}_{3}\right) \in$ $\left[L^{2}\left(\Omega_{k}\right)\right]^{3} \times\left[L^{2}\left(\Omega_{k}\right)\right]^{3} \times\left[L^{2}\left(\Omega_{k}\right)\right]^{3}$.

By the expression of the scalar variables $\mathbf{v}=\left(v_{1}, v_{2}, v_{3}\right)$, we introduce the inequality, $\sum_{i} \frac{1}{\kappa} \int_{\Omega_{k}}\left(\mathbf{q}_{i}^{ \pm}-\right.$ $\left.\kappa \nabla v_{i}\right)^{2} d \Omega \geq 0$, which can be rewritten as $\kappa\left\{a_{\Omega_{k}}(\mathbf{v}, \mathbf{v})\right\} \geq-\frac{1}{\kappa} \int_{\Omega_{k}} \mathbf{q}_{i}^{ \pm} \cdot \mathbf{q}_{i}^{ \pm} d \Omega+2 \int_{\Omega_{k}} \mathbf{q}_{i}^{ \pm} \cdot \nabla v_{i} d \Omega$. Note that the repeated indices are summed unless specified otherwisely. We can now write the inequality related to the Lagrangian (53):

$$
\begin{aligned}
\mathcal{L}_{\Omega_{k}}^{ \pm}\left(\mathbf{v}, \beta, \pm \psi_{h}, \pm \theta_{h}, \kappa \mathbf{t}_{h}^{p r} \pm \mathbf{t}_{h}^{d u}\right) \geq & -\frac{1}{\kappa} \int_{\Omega_{k}} \mathbf{q}_{i}^{ \pm} \cdot \mathbf{q}_{i}^{ \pm} d \Omega+2 \int_{\Omega_{k}} \mathbf{q}_{i}^{ \pm} \cdot \nabla v_{i} d \Omega \\
& +\kappa\left\{a_{\Omega_{k}}\left(\mathbf{u}_{h}, \mathbf{v}\right)+b_{\Omega_{k}}\left(\mathbf{v}, p_{h}\right)-\ell_{\Omega_{k}}^{N}(\mathbf{v})+H_{\Omega_{k}}\left(\mathbf{v}, \mathbf{t}_{h}^{p r}\right)\right\} \\
& \pm\left\{a_{\Omega_{k}}\left(\mathbf{v}, \psi_{h}\right)+b_{\Omega_{k}}\left(\mathbf{v}, \theta_{h}\right)+\ell_{\Omega_{k}}^{0}(\mathbf{v})+H_{\Omega_{k}}\left(\mathbf{v}, \mathbf{t}_{h}^{d u}\right)\right\} \\
& \pm\left\{\ell_{\Omega_{k}}^{0}\left(\mathbf{u}_{h}\right)+a_{\Omega_{k}}\left(\mathbf{u}_{h}, \psi_{h}\right)+b_{\Omega_{k}}\left(\psi_{h}, p_{h}\right)-\ell_{\Omega_{k}}^{N}\left(\psi_{h}\right)+b_{\Omega_{k}}\left(\mathbf{u}_{h}, \theta_{h}\right)\right\} \\
& +\kappa\left\{2 b_{\Omega_{k}}(\mathbf{v}, \beta)+b_{\Omega_{k}}\left(\mathbf{u}_{h}, \beta\right)\right\} \pm b_{\Omega_{k}}\left(\psi_{h}, \beta\right)
\end{aligned}
$$

The right hand side of (61) is an elemental contribution to the bound that, depending on the sign, contributes to the lower and upper bounds to the exact output.

From the inequality (61) we define a new augmented Lagrangian $\mathcal{J}_{\Omega_{k}}^{ \pm}\left(\mathbf{q}^{ \pm}, \beta, \mathbf{v}\right)$ with the variables, $\beta=\epsilon^{ \pm}$and $\mathbf{v}=\zeta^{ \pm}$, corresponding to the pressure and velocity perturbations, respectively:

$$
\begin{aligned}
& \mathcal{J}_{\Omega_{k}}^{ \pm}\left(\mathbf{q}^{ \pm}, \epsilon^{ \pm}, \zeta^{ \pm}\right) \\
&=-\frac{1}{\kappa} \int_{\Omega_{k}} \mathbf{q}_{i}^{ \pm} \cdot \mathbf{q}_{i}^{ \pm} d \Omega+2 \int_{\Omega_{k}} \mathbf{q}_{i}^{ \pm} \cdot \nabla \zeta^{ \pm} d \Omega+\kappa\left\{2 b_{\Omega_{k}}\left(\zeta^{ \pm}, \epsilon^{ \pm}\right)+b_{\Omega_{k}}\left(\mathbf{u}_{h}, \epsilon^{ \pm}\right)\right\} \pm b_{\Omega_{k}}\left(\psi_{h}, \epsilon^{ \pm}\right) \\
&+\kappa\left\{a_{\Omega_{k}}\left(\mathbf{u}_{h}, \zeta^{ \pm}\right)+b_{\Omega_{k}}\left(\zeta^{ \pm}, p_{h}\right)-\ell_{\Omega_{k}}^{N}\left(\zeta^{ \pm}\right)+H_{\Omega_{k}}\left(\zeta^{ \pm}, t_{h}^{p r}\right)\right\} \\
& \pm\left\{a_{\Omega_{k}}\left(\zeta^{ \pm}, \psi_{h}\right)+b_{\Omega_{k}}\left(\zeta^{ \pm}, \theta_{h}\right)+\ell_{\Omega_{k}}^{0}\left(\zeta^{ \pm}\right)+H_{\Omega_{k}}\left(\zeta^{ \pm}, t_{h}^{d u}\right)\right\} \\
& \pm\left\{\ell_{\Omega_{k}}^{0}\left(\mathbf{u}_{h}\right)+a_{\Omega_{k}}\left(\mathbf{u}_{h}, \psi_{h}\right)+b_{\Omega_{k}}\left(\psi_{h}, p_{h}\right)-\ell_{\Omega_{k}}^{N}\left(\psi_{h}\right)+b\left(\mathbf{u}_{h}, \theta_{h}\right)\right\}
\end{aligned}
$$

To find the saddle point of the augmented Lagrangian (62), the functions denoted by $\mathbf{q}^{ \pm}+\mathbf{g}$ are substituted into the Lagrangian. That the variation of $\mathcal{J}_{\Omega_{k}}^{ \pm}$with respect to $\mathbf{g}$ vanishes leads to

$$
\mathcal{J}_{\Omega_{k}}^{ \pm}\left(\mathbf{q}^{ \pm}+\mathbf{g}, \epsilon^{ \pm}, \zeta^{ \pm}\right)-\mathcal{J}_{\Omega_{k}}^{ \pm}\left(\mathbf{q}^{ \pm}, \epsilon^{ \pm}, \zeta^{ \pm}\right)=0, \text { with } \mathbf{g} \rightarrow 0,
$$

or

$$
-\frac{2}{\kappa} \int_{\Omega_{k}} \mathbf{q}_{i}^{ \pm} \cdot \mathbf{g}_{i} d \Omega+2 \int_{\Omega_{k}} \mathbf{g}_{i} \cdot \nabla \zeta_{i}^{ \pm} d \Omega=0, \quad \forall\left(\mathbf{g}_{1}, \mathbf{g}_{2}, \mathbf{g}_{3}\right) \in\left[L^{2}\left(\Omega_{k}\right)\right]^{3} \times\left[L^{2}\left(\Omega_{k}\right)\right]^{3} \times\left[L^{2}\left(\Omega_{k}\right)\right]^{3} .
$$


Similarly, the variation of $\mathcal{J}_{\Omega_{k}}^{ \pm}$with $\beta$ vanishes: $\mathcal{J}_{\Omega_{k}}^{ \pm}\left(\mathbf{q}^{ \pm}, \epsilon^{ \pm}+\beta, \zeta^{ \pm}\right)-\mathcal{J}_{\Omega_{k}}^{ \pm}\left(\mathbf{q}^{ \pm}, \epsilon^{ \pm}, \zeta^{ \pm}\right)=0$ which leads to

$$
\kappa\left\{2 b_{\Omega_{k}}\left(\zeta^{ \pm}, \beta\right)+b_{\Omega_{k}}\left(\mathbf{u}_{h}, \beta\right)\right\} \pm b_{\Omega_{k}}\left(\psi_{h}, \beta\right)=0, \quad \forall \beta \in L^{2}\left(\Omega_{k}\right) .
$$
to

The variation of $\mathcal{J}_{\Omega_{k}}^{ \pm}$with $\mathbf{v}$ vanishes: $\mathcal{J}_{\Omega_{k}}^{ \pm}\left(\mathbf{q}^{ \pm}, \epsilon^{ \pm}, \zeta^{ \pm}+\mathbf{v}\right)-\mathcal{J}_{\Omega_{k}}^{ \pm}\left(\mathbf{q}^{ \pm}, \epsilon^{ \pm}, \zeta^{ \pm}\right)=0$, which leads

$$
\begin{array}{r}
2 \int_{\Omega_{k}} \mathbf{q}_{i}^{ \pm} \cdot \nabla v_{i} d \Omega+\kappa\left\{a_{\Omega_{k}}\left(\mathbf{u}_{h}, \mathbf{v}\right)+b\left(\mathbf{v}, p_{h}\right)-\ell_{\Omega_{k}}^{N}(\mathbf{v})+H_{\Omega_{k}}\left(\mathbf{v}, \mathbf{t}_{h}^{p r}\right)\right\} \\
\pm\left\{a_{\Omega_{k}}\left(\mathbf{v}, \psi_{h}\right)+b_{\Omega_{k}}\left(\mathbf{v}, \theta_{h}\right)+\ell_{\Omega_{k}}^{0}(\mathbf{v})+H_{\Omega_{k}}\left(\mathbf{v}, \mathbf{t}_{h}^{d u}\right)\right\}+\kappa\left\{2 b_{\Omega_{k}}\left(\mathbf{v}, \epsilon^{ \pm}\right)\right\}=0, \\
\forall\left(v_{1}, v_{2}, v_{3}\right) \in\left[L^{2}\left(\Omega_{k}\right)\right]^{3} .
\end{array}
$$

By definition $\mathbf{q}^{ \pm}=\kappa \mathbf{q}^{p r} \pm \mathbf{q}^{d u}, \epsilon^{ \pm}=\epsilon^{p r} \pm \frac{1}{\kappa} \epsilon^{d u}$ and $\zeta^{ \pm}=\zeta^{p r} \pm \frac{1}{\kappa} \zeta^{d u}$, equations (64) (65) and (66) can be expressed without $\kappa$ : find $\mathbf{q}^{p r} \in\left[L^{2}\left(\Omega_{k}\right)\right]^{3} \times\left[L^{2}\left(\Omega_{k}\right)\right]^{3} \times\left[L^{2}\left(\Omega_{k}\right)\right]^{3}, \mathbf{q}^{d u} \in$ $\left[L^{2}\left(\Omega_{k}\right)\right]^{3} \times\left[L^{2}\left(\Omega_{k}\right)\right]^{3} \times\left[L^{2}\left(\Omega_{k}\right)\right]^{3}, \epsilon^{p r} \in L^{2}\left(\Omega_{k}\right), \epsilon^{d u} \in L^{2}\left(\Omega_{k}\right), \zeta^{p r} \in\left[L^{2}\left(\Omega_{k}\right)\right]^{3}$ and $\zeta^{d u} \in\left[L^{2}\left(\Omega_{k}\right)\right]^{3}$, such that:

$$
\begin{aligned}
2\left\{-\int_{\Omega_{k}} \mathbf{q}_{i}^{p r} \cdot \mathbf{g}_{i} d \Omega+\int_{\Omega_{k}} \mathbf{g}_{i} \cdot \nabla \zeta_{i}^{p r} d \Omega\right\}= & 0, \forall\left(\mathbf{g}_{1}, \mathbf{g}_{2}, \mathbf{g}_{3}\right) \in\left[L^{2}\left(\Omega_{k}\right)\right]^{3} \times\left[L^{2}\left(\Omega_{k}\right)\right]^{3} \times\left[L^{2}\left(\Omega_{k}\right)\right]^{3} ; \\
2\left\{-\int_{\Omega_{k}} \mathbf{q}_{i}^{d u} \cdot \mathbf{g}_{i} d \Omega+\int_{\Omega_{k}} \mathbf{g}_{i} \cdot \nabla \zeta_{i}^{d u} d \Omega\right\}= & 0, \forall\left(\mathbf{g}_{1}, \mathbf{g}_{2}, \mathbf{g}_{3}\right) \in\left[L^{2}\left(\Omega_{k}\right)\right]^{3} \times\left[L^{2}\left(\Omega_{k}\right)\right]^{3} \times\left[L^{2}\left(\Omega_{k}\right)\right]^{3} ; \\
2 b_{\Omega_{k}}\left(\zeta^{p r}, \beta\right)= & -b_{\Omega_{k}}\left(\mathbf{u}_{h}, \beta\right), \quad \forall \beta \in L^{2}\left(\Omega_{k}\right) ; \\
2 b_{\Omega_{k}}\left(\zeta^{d u}, \beta\right)= & -b_{\Omega_{k}}\left(\psi_{h}, \beta\right), \quad \forall \beta \in L^{2}\left(\Omega_{k}\right) ; \\
2\left\{\int_{\Omega_{k}} \mathbf{q}^{p r} \cdot \nabla v_{i} d \Omega+b_{\Omega_{k}}\left(\mathbf{v}, \epsilon^{p r}\right)\right\}= & -\left\{a_{\Omega_{k}}\left(\mathbf{u}_{h}, \mathbf{v}\right)+b_{\Omega_{k}}\left(\mathbf{v}, p_{h}\right)-\ell_{\Omega_{k}}^{N}(\mathbf{v})+H_{\Omega_{k}}\left(\mathbf{v}, \mathbf{t}_{h}^{p r}\right)\right\}, \\
2\left\{\int_{\Omega_{k}} \mathbf{q}^{d u} \cdot \nabla v_{i} d \Omega+b_{\Omega_{k}}\left(\mathbf{v}, \epsilon^{d u}\right)\right\}= & \mp\left\{a_{\Omega_{k}}\left(\mathbf{v}, \psi_{h}\right)+b_{\Omega_{k}}\left(\mathbf{v}, \theta_{h}\right)+\ell_{\Omega_{k}}^{0}(\mathbf{v})+H_{\Omega_{k}}\left(\mathbf{v}, \mathbf{t}_{h}^{d u}\right)\right\}, \\
& \forall\left(v_{1}, v_{2}, v_{3}\right) \in\left[L^{2}\left(\Omega_{k}\right)\right]^{3} .
\end{aligned}
$$

Equations (97) and (98) in Section 4.5 show the matrix systems used for solving the above equations (67) to (72).

\subsection{Bounds}

Equations (64), (65) and (66) are substituted into the new augmented Lagrangian (62) to calculate the elemental contributions to the bounds. Note that in equation (61), the right-hand side bounds from below, therefore a finite element solution of the above equations will bound from below as the quadratic term is $-\int_{\Omega_{k}} \mathbf{q}_{i}^{ \pm} \cdot \mathbf{q}_{i}^{ \pm} d \Omega$. By introducing the subscript $h$ for a finite element solution of equations (67)-(72), we can now calculate $\eta_{h}^{ \pm}$as described below.

From (64), we have $-\frac{2}{\kappa} \int_{\Omega_{k}} \mathbf{q}_{i h}^{ \pm} \cdot \mathbf{q}_{i h}^{ \pm} d \Omega+2 \int_{\Omega_{k}} \mathbf{q}_{i h}^{ \pm} \cdot \nabla \zeta_{i h}^{ \pm} d \Omega=0$. From (65), we have $\kappa\left\{2 b_{\Omega_{k}}\left(\zeta_{h}^{ \pm}, \epsilon^{ \pm}\right)+b_{\Omega_{k}}\left(\mathbf{u}_{h}, \epsilon^{ \pm}\right)\right\} \pm b_{\Omega_{k}}\left(\psi_{h}, \epsilon^{ \pm}\right)=0$. From (66), we have $2 \int_{\Omega_{k}} \mathbf{q}_{i h}^{ \pm} \cdot \nabla \zeta_{i h}^{ \pm} d \Omega+$ $\kappa\left\{a_{\Omega_{k}}\left(\mathbf{u}_{h}, \zeta_{h}^{ \pm}\right)+b_{\Omega_{k}}\left(\zeta_{h}^{ \pm}, p_{h}\right)-\ell_{\Omega_{k}}^{N}\left(\zeta_{h}^{ \pm}\right)+H_{\Omega_{k}}\left(\zeta_{h}^{ \pm}, \mathbf{t}_{h}^{p r}\right)\right\} \pm\left\{a_{\Omega_{k}}\left(\zeta_{h}^{ \pm}, \psi_{h}\right)+b_{\Omega_{k}}\left(\zeta_{h}^{ \pm}, \theta_{h}\right)+\ell_{\Omega_{k}}^{0}\left(\zeta_{h}^{ \pm}\right)+\right.$ $\left.H_{\Omega_{k}}\left(\zeta_{h}^{ \pm}, \mathbf{t}_{h}^{d u}\right)\right\}+\kappa\left\{2 b_{\Omega_{k}}\left(\zeta_{h}^{ \pm}, \epsilon^{ \pm}\right)\right\}=0$. Therefore, the bounds can be written as: 


$$
\begin{aligned}
\eta_{h}^{ \pm}= & \sum_{\Omega_{k}}\left(-\frac{1}{\kappa} \int_{\Omega_{k}} \mathbf{q}_{i h}^{ \pm} \cdot \mathbf{q}_{i h}^{ \pm} d \Omega-\kappa\left\{2 b_{\Omega_{k}}\left(\zeta_{h}^{ \pm}, \epsilon^{ \pm}\right)\right\}\right. \\
& \left. \pm\left\{\ell_{\Omega_{k}}^{0}\left(\mathbf{u}_{h}\right)+a_{\Omega_{k}}\left(\mathbf{u}_{h}, \psi_{h}\right)+b_{\Omega_{k}}\left(\psi_{h}, p_{h}\right)-\ell_{\Omega_{k}}^{N}\left(\psi_{h}\right)+b_{\Omega_{k}}\left(\mathbf{u}_{h}, \theta_{h}\right)\right\}\right) \\
= & \sum_{\Omega_{k}}\left(-\frac{1}{\kappa} \int_{\Omega_{k}} \mathbf{q}_{i h}^{ \pm} \cdot \mathbf{q}_{i h}^{ \pm} d \Omega-\kappa\left\{2 b_{\Omega_{k}}\left(\zeta_{h}^{ \pm}, \epsilon^{ \pm}\right)\right\}+\mathbf{C}_{\Omega_{k}}^{ \pm}\right) \\
= & \sum_{\Omega_{k}}\left(-\kappa \int_{\Omega_{k}} \mathbf{q}_{i h}^{p r} \cdot \mathbf{q}_{i h}^{p r} d \Omega-\frac{1}{\kappa} \int_{\Omega_{k}} \mathbf{q}_{i h}^{d u} \cdot \mathbf{q}_{i h}^{d u} d \Omega \mp 2 \int_{\Omega_{k}} \mathbf{q}_{i h}^{d u} \cdot \mathbf{q}_{i h}^{p r} d \Omega\right. \\
& -\kappa\left\{2 b_{\Omega_{k}}\left(\zeta_{h}^{p r}, \epsilon^{p r}\right)\right\} \\
& \left.-\frac{1}{\kappa}\left\{2 b_{\Omega_{k}}\left(\zeta_{h}^{d u}, \epsilon^{d u}\right)\right\} \mp\left\{2 b_{\Omega_{k}}\left(\zeta_{h}^{p r}, \epsilon^{d u}\right)\right\} \mp\left\{2 b_{\Omega_{k}}\left(\zeta_{h}^{d u}, \epsilon^{p r}\right)\right\}+\mathbf{C}_{\Omega^{k}}^{ \pm}\right)
\end{aligned}
$$

By defining

$$
\begin{aligned}
z_{i}^{p r} & =\sum_{\Omega_{k}} \int_{\Omega_{k}} \mathbf{q}_{i h}^{p r} \cdot \mathbf{q}_{i h}^{p r} d \Omega \\
z_{i}^{d u} & =\sum_{\Omega_{k}} \int_{\Omega_{k}} \mathbf{q}_{i h}^{d u} \cdot \mathbf{q}_{i h}^{d u} d \Omega \\
\bar{z}_{i} & =\sum_{\Omega_{k}} \int_{\Omega_{k}} \mathbf{q}_{i h}^{p r} \cdot \mathbf{q}_{i h}^{d u} d \Omega \\
b_{11} & =\sum_{\Omega_{k}} b_{\Omega_{k}}\left(\zeta_{h}^{p r}, \epsilon^{p r}\right), \\
b_{22} & =\sum_{\Omega_{k}} b_{\Omega_{k}}\left(\zeta_{h}^{d u}, \epsilon^{d u}\right), \\
b_{12} & =\sum_{\Omega_{k}} b_{\Omega_{k}}\left(\zeta_{h}^{p r}, \epsilon^{d u}\right), \\
b_{21} & =\sum_{\Omega_{k}} b_{\Omega_{k}}\left(\zeta_{h}^{d u}, \epsilon^{p r}\right), \\
\mathbf{C}^{ \pm} & =\sum_{\Omega_{k}} \mathbf{C}_{\Omega_{k}}^{ \pm}
\end{aligned}
$$

we can express (73) as

$$
\eta_{h}^{ \pm}=-\kappa z_{i}^{p r}-\frac{1}{\kappa} z_{i}^{d u} \mp 2 \bar{z}_{i}-2 \kappa b_{11}-\frac{2}{\kappa} b_{22} \mp 2 b_{12} \mp 2 b_{21}+\mathbf{C}^{ \pm} .
$$

$\kappa$ can be optimized by solving

$$
\frac{\partial}{\partial \kappa}\left\{-\kappa z_{i}^{p r}-\frac{1}{\kappa} z_{i}^{d u} \mp 2 \bar{z}_{i}-2 \kappa b_{11}-\frac{2}{\kappa} b_{22} \mp 2 b_{12} \mp 2 b_{21}+\mathbf{C}^{ \pm}\right\}=0,
$$

which leads to

$$
\kappa=\sqrt{\frac{z_{i}^{d u}+2 b_{22}}{z_{i}^{p r}+2 b_{11}}} .
$$


Therefore the optimized bounds are:

$$
\begin{aligned}
\eta_{h}^{ \pm} & =\mathbf{C}^{ \pm} \mp 2\left(\bar{z}_{i}+b_{12}+b_{21}\right)-2 \sqrt{\left(z_{i}^{d u}+2 b_{22}\right)\left(z_{i}^{p r}+2 b_{11}\right)} \\
s_{h}^{+}=-\eta_{h}^{-} & =-\mathbf{C}^{-}-2\left(\bar{z}_{i}+b_{12}+b_{21}\right)+2 \sqrt{\left(z_{i}^{d u}+2 b_{22}\right)\left(z_{i}^{p r}+2 b_{11}\right)} \\
s_{h}^{-}=\eta_{h}^{+} & =\mathbf{C}^{+}-2\left(\bar{z}_{i}+b_{12}+b_{21}\right)-2 \sqrt{\left(z_{i}^{d u}+2 b_{22}\right)\left(z_{i}^{p r}+2 b_{11}\right)}
\end{aligned}
$$

or

$$
\begin{aligned}
s_{h}^{+} & =\tilde{s}_{h}+2 \sqrt{\left(z_{i}^{d u}+2 b_{22}\right)\left(z_{i}^{p r}+2 b_{11}\right)} \\
& =\tilde{s}_{h}+2 \sqrt{\left\{\int \mathbf{q}_{i h}^{p r} \cdot \mathbf{q}_{i h}^{p r} d \Omega+2 b\left(\zeta_{h}^{p r}, \epsilon^{p r}\right)\right\}\left\{\int \mathbf{q}_{i h}^{d u} \cdot \mathbf{q}_{i h}^{d u} d \Omega+2 b\left(\zeta_{h}^{d u}, \epsilon^{d u}\right)\right\}} \\
s_{h}^{-} & =\tilde{s}_{h}-2 \sqrt{\left(z_{i}^{d u}+2 b_{22}\right)\left(z_{i}^{p r}+2 b_{11}\right)} \\
& =\tilde{s}_{h}-2 \sqrt{\left\{\int \mathbf{q}_{i h}^{p r} \cdot \mathbf{q}_{i h}^{p r} d \Omega+2 b\left(\zeta_{h}^{p r}, \epsilon^{p r}\right)\right\}\left\{\int \mathbf{q}_{i h}^{d u} \cdot \mathbf{q}_{i h}^{d u} d \Omega+2 b\left(\zeta_{h}^{d u}, \epsilon^{d u}\right)\right\}}
\end{aligned}
$$

where $\tilde{s}_{h}$ is the average of the upper and lower bounds $\tilde{s}_{h}=\frac{1}{2}\left(s_{h}^{+}+s_{h}^{-}\right)$:

$$
\tilde{s}_{h}= \pm \mathbf{C}^{ \pm}-2\left(\bar{z}_{i}+b_{12}+b_{21}\right) \text {. }
$$

\section{The Discrete Bound Procedure}

The exact bound procedure for the Stokes problem is now expressed in a matrix formulation. The first three steps are to inexpensively calculate the field solution and the Lagrange multiplier candidates: adjoint and hybrid flux. These calculations are performed on the mesh $\mathcal{T}_{h}$. The last step requires calculations of the local high order discretization problems on $\Omega^{k}$.

\subsection{The primal problem}

The primal problem is the original Stokes problem expressed by equations (34) and (35). The resulting primal problem is trivial, reflecting the step to find the global field solution of the Stokes equation: Find $\left[u_{1 h}, u_{2 h}, u_{3 h}, p_{h}\right]^{T} \in X_{h}^{\text {low }} \times \mathcal{Q}_{h}$, such that

$$
\left[\begin{array}{cccc}
A_{h} & & & B_{1 h}^{T} \\
& A_{h} & & B_{2 h}^{T} \\
& & A_{h} & B_{3 h}^{T} \\
B_{1 h} & B_{2 h} & B_{3 h} &
\end{array}\right]\left[\begin{array}{c}
u_{1 h} \\
u_{2 h} \\
u_{3 h} \\
p_{h}
\end{array}\right]=\left[\begin{array}{c}
M_{h} f_{1 h} \\
M_{h} f_{2 h} \\
M_{h} f_{3 h} \\
0
\end{array}\right] .
$$

Here $A_{h}$ is the stiffness matrix, $M_{h}$ the mass matrix and $B_{h}$ the matrix related to the pressure. All the matrices are constructed for the lower order approximation.

\subsection{The dual problem}

The dual problem is obtained from equations (34) and (35), that is to find the adjoint solution $\left[\psi_{1 h}, \psi_{2 h}, \psi_{3 h}, \lambda_{h}\right]^{T} \in X_{h}^{\text {low }} \times \mathcal{Q}_{h}$, such that

$$
\left[\begin{array}{cccc}
A_{h} & & & B_{1 h}^{T} \\
& A_{h} & & B_{2 h}^{T} \\
& & A_{h} & B_{3 h}^{T} \\
B_{1 h} & B_{2 h} & B_{3 h} &
\end{array}\right]\left[\begin{array}{c}
\psi_{1 h} \\
\psi_{2 h} \\
\psi_{3 h} \\
\lambda_{h}
\end{array}\right]=-\left[\begin{array}{c}
0 \\
0 \\
M_{h} 1 \\
0
\end{array}\right] .
$$




\subsection{The hybrid flux problem}

The hybrid flux vector, $\mathbf{t}_{h}^{ \pm}=\kappa \mathbf{t}_{h}^{p r} \pm \mathbf{t}_{h}^{d u}$, is the Lagrangian multiplier that enforces the intersubdomain continuity, and can be evaluated with two residual equations, one from decoupling the primal problem and the other from decoupling the dual problem onto the subdomains. Note that $\mathbf{t}_{h}^{ \pm}$is calculated in $\mathbf{E}_{h}^{\text {low }}$ and then interpolated on $\mathbf{E}_{h}$.

\subsubsection{The hybrid flux associated with the primal problem}

The hybrid flux problem corresponding to the primal problem is to solve the following algebraic equation:

$$
\left\{\begin{array}{cl}
2 A_{h}^{(k)} e_{i h}^{p r(k)}+A_{h}^{(k)} u_{i h}^{(k)}+B_{i h}^{(k)^{T}} p_{h}^{(k)}-M_{h}^{(k)} f_{i h}^{(k)}+h_{h}^{(k)^{T}} t_{i h}^{p r} & =0, \\
\sum_{k=1}^{N_{k}} H_{h}^{(k)} e_{i h}^{p r(k)} & =0,
\end{array}\right.
$$

where $H_{h}^{(k)}$ is the boolean matrix related to the hybrid flux edge space. Note that the superscript $*^{(k)}$ denotes the decoupled subdomain with index $k$. Note that $B_{i h}^{(k)} u_{i h}^{(k)}=B_{i h}^{(k)} e_{i h}^{p r(k)}=0$ on the global mesh. The above equation can be inexpensively calculated by the directly equilibrated hybrid-flux approach [8]:

$$
H_{h}^{(k)} H_{h}^{(k)^{T}} t_{i h}^{p r}=H_{h}^{(k)}\left(M_{h}^{(k)} f_{i h}^{(k)}-\left[A_{h}^{(k)} u_{i h}^{(k)}+B_{i h}^{(k)^{T}} p_{h}^{(k)}\right]\right)
$$

\subsubsection{The hybrid flux associated with the dual problem}

Similarly, the hybrid flux equation for the dual problem $t_{i H}^{d u}$ is

$$
\left\{\begin{array}{cl}
2 A_{h}^{(k)} e_{i h}^{d u(k)}+A_{h}^{(k)} \psi_{i h}^{(k)}+B_{i h}^{(k)^{T}} \lambda_{h}^{(k)}+M_{h}^{(k)} f_{i h}^{0(k)}+H_{h}^{(k)^{T}} t_{i h}^{d u} & =0 \\
\sum_{k=1}^{N_{k}} H_{h}^{(k)} e_{i h}^{d u(k)} & =0
\end{array}\right.
$$

where $f_{1 h}^{0(k)}=f_{2 h}^{0(k)}=0$ and $f_{3 h}^{0(k)}=\ell_{3 h}^{(k)}=1$.

It can be calculated by

$$
H_{h}^{(k)} H_{h}^{(k)^{T}} t_{i h}^{d u}=H_{h}^{(k)}\left(M_{h}^{(k)} 1_{i h}^{(k)}+A_{h}^{(k)} \psi_{i h}^{(k)}+B_{i h}^{(k)^{T}} \lambda_{h}^{(k)}\right)
$$

\subsection{Local calculations}

To start, all the variables $u_{i h}^{(k)}, p_{h}^{(k)}, \psi_{i h}^{(k)}, \lambda_{h}^{(k)}, t_{i h}^{p r}$ and $t_{i h}^{d u}$ are interpolated from the low order approximation to the higher order approximation $\hat{X}_{h}$.

\subsection{Subdomain error estimation calculations}

The primal decoupled subdomain error estimation problem for equations (67), (69) and (71) are written discretely as: 


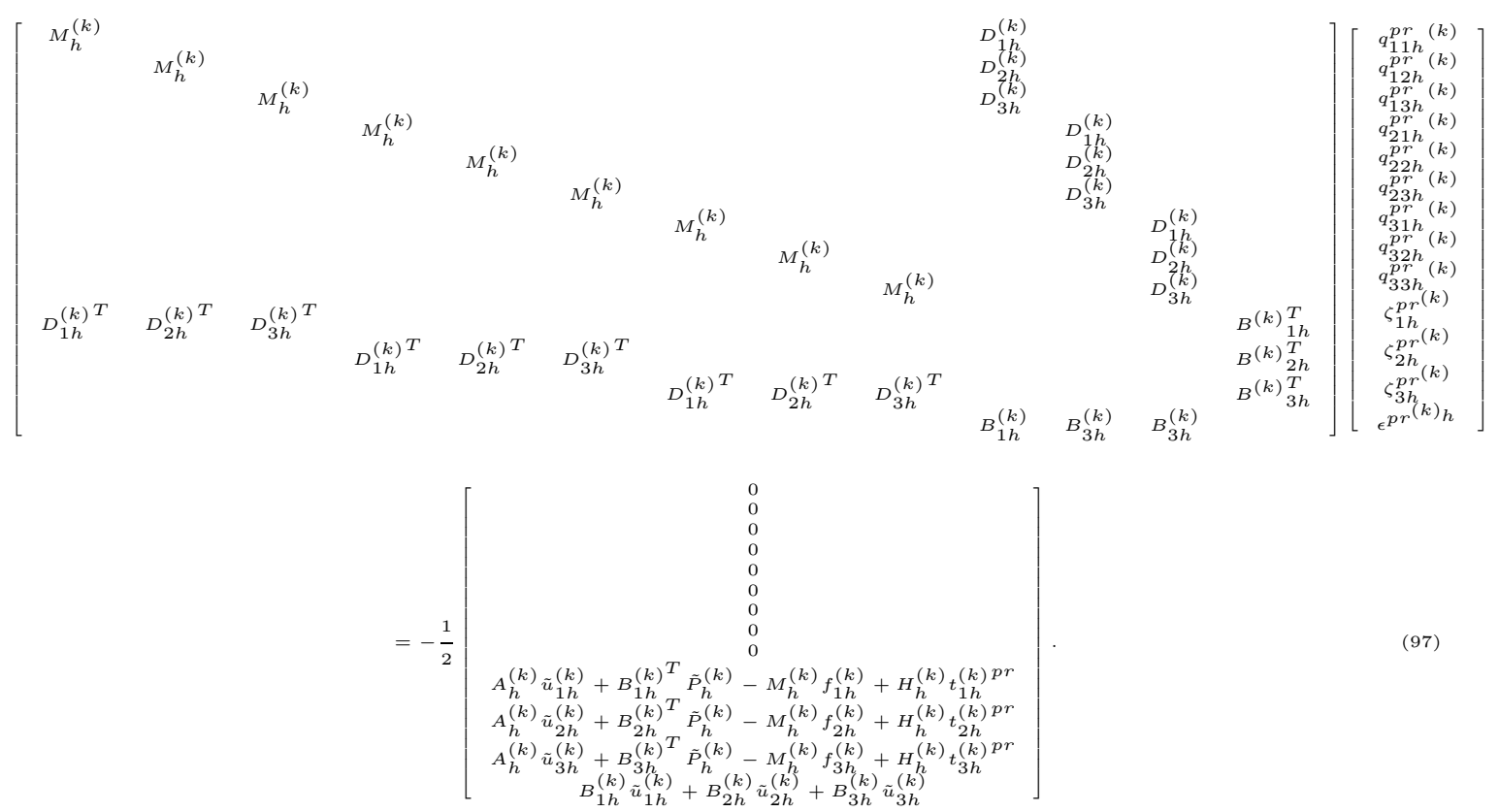

The dual decoupled subdomain error estimation problem for equations (68), (70) and (72) are written discretely as:

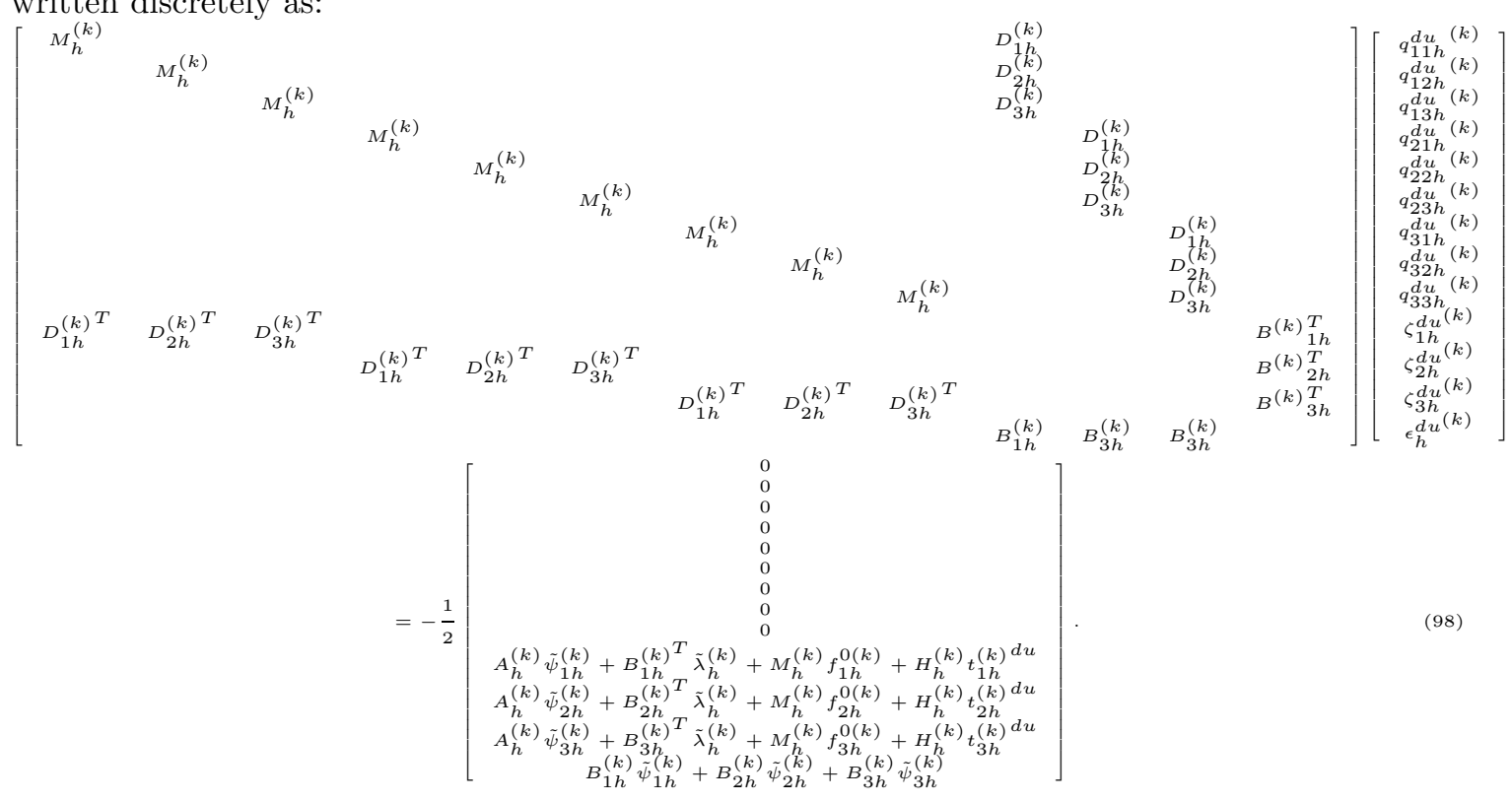




\subsection{The lower and upper bounds}

We can write $z^{p r}, z^{d u}, \bar{z}$, and $b_{i j}(i, j=1,2)$ discretely as functions of $\hat{\mathbf{q}}_{i h}^{ \pm}, \epsilon_{h}^{ \pm}$and $\zeta_{i h}^{ \pm}$,:

$$
\begin{aligned}
z^{p r}=\sum_{k=1}^{N_{k}} \sum_{i=1}^{3} \sum_{j=1}^{3}\left[q_{i j h}^{p r}{ }^{(k)} M_{h}^{(k)} q_{i j h}^{p r}{ }^{(k)}\right], & \bar{z}=\sum_{k=1}^{N_{k}} \sum_{i=1}^{3} \sum_{j=1}^{3}\left[q_{i j h}^{p r}{ }^{(k)} M_{h}^{(k)} q_{i j h}^{d u}{ }^{(k)}\right], \\
z^{d u}=\sum_{k=1}^{N_{k}} \sum_{i=1}^{3} \sum_{j=1}^{3}\left[q_{i j h}^{d u}{ }^{(k)} M_{h}^{(k)} q_{i j h}^{d u}{ }^{(k)}\right], & \\
b_{11}=\sum_{k=1}^{N_{k}} \sum_{i=1}^{3} \epsilon_{h}^{p r^{(k)}} B_{i h}^{(k)} \zeta_{i h}^{p r^{(k)}}, & b_{12}=\sum_{k=1}^{N_{k}} \sum_{i=1}^{3} \epsilon_{h}^{d u^{(k)}} B_{i h}^{(k)} \zeta_{i h}^{p r^{(k)}}, \\
b_{21}=\sum_{k=1}^{N_{k}} \sum_{i=1}^{3} \epsilon_{h}^{p r^{(k)}} B_{i h}^{(k)} \zeta_{i}^{d u^{(k)}}, & b_{22}=\sum_{k=1}^{N_{k}} \sum_{i=1}^{3} \epsilon_{h}^{d u^{(k)}} B_{i h}^{(k)} \zeta_{i h}^{d u^{(k)}} .
\end{aligned}
$$

Once the computed values for $\hat{\mathbf{q}}_{i h}^{ \pm}, \epsilon_{h}^{ \pm}$and $\zeta_{i h}^{ \pm}$are inserted into the Lagrangian (62), the optimized upper and lower bounds follow the bounding properties and can be expressed in matrix forms as:

$$
\begin{aligned}
& s_{h}^{-}=\tilde{s}_{h}-2 \sqrt{\left(z_{i}^{d u}+2 b_{22}\right)\left(z_{i}^{p r}+2 b_{11}\right)} \\
& s_{h}^{+}=\tilde{s}_{h}+2 \sqrt{\left(z_{i}^{d u}+2 b_{22}\right)\left(z_{i}^{p r}+2 b_{11}\right)}
\end{aligned}
$$

where the average of the lower and upper bounds is

$$
\begin{aligned}
\tilde{s}_{h}= & \sum_{i=1}^{3} u_{i h}^{(k) T} M_{h}^{(k)} 1+\sum_{i=1}^{3} \sum_{k=1}^{N_{k}}\left[u_{i h}^{(k) T} A_{h}^{(k)} \psi_{i h}^{(k)}+p_{h}^{(k) T} B_{i h}^{(k)} \psi_{i h}^{(k)}+\lambda_{h}^{(k) T} B_{i h}^{(k)} u_{i h}^{(k)}-f_{h}^{(k)^{T}} M_{h}^{(k)} \psi_{i h}^{(k)}\right] \\
& -2 \bar{z}-2 b_{12}-2 b_{21} .
\end{aligned}
$$

Note that the bound gap, given by:

$$
\Delta=\left|s_{h}^{+}-s_{h}^{-}\right|=4 \sqrt{\left(z_{i}^{d u}+2 b_{22}\right)\left(z_{i}^{p r}+2 b_{11}\right)}
$$

is made of positive contributions from each element so we can define $\Delta^{(k)}$ as a bound contribution in each element and used it in an adaptive mesh refinement strategy.

\section{Numerical Example of Bound Calculations for Stokes Out- put}

Figure 8 shows the numerical results of the velocity field for the Stokes problem at three sections defined by $x_{1}=0.25, x_{1}=0.5$ and $x_{1}=0.75$.

Table 2 shows the lower bound $\left(s_{h}^{-}\right)$, upper bound $\left(s_{h}^{+}\right)$, the average of the bounds $\left(s_{h}^{\text {pre }}\right)$ and discrete output to the solution $\left(s_{h}\right)$ obtained on the mesh size $h$ using the Exact Stokes Bounds method. For the finest mesh, a half bound gap of $7.1 \%$ is reached. The half bound gap is the bound gap divided by 2 and normalized with with the most accurate output value. Note that the error between the finite element output value on the finest mesh and the most accurate output values is $3.1 \%$ which shows that the bounds are quite sharp and just over-predict the error by a 

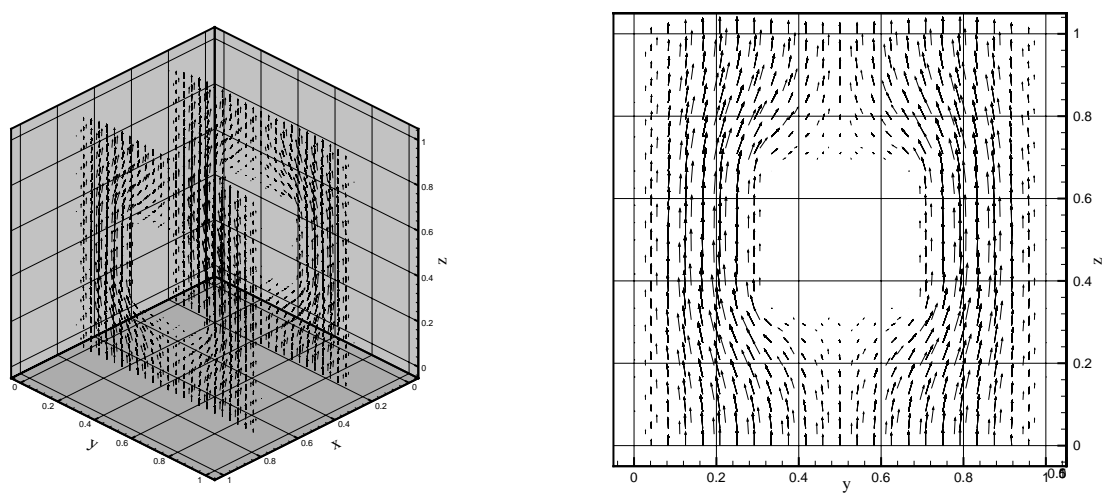

Figure 8: Velocity profile for the Stokes problem with the periodic boundary conditions (left) velocity at $x_{1}=0.25$ and $x_{1}=0.75$; (right) velocity at $x_{1}=0.5$.

factor of two. The most accurate output value $\tilde{s}=0.007283$ is obtained on the Crouzeix-Raviart finite element space with mesh size $h=0.02$. Note that for a relative coarse mesh (number of elements per side $N=12, N_{k}=6 \times N^{3}$ ) the bound gap is quite large with a half bound gap of $22.5 \%$ but so is the finite element output on this mesh which has an error of $11.8 \%$. The same problem was examined with an hierarchical approach in [6]. Note that the hierarchical approach bounds a fine mesh output and not the output of the weak finite element solution. For these calculations a refinement of three $(R=3)$ was used which means that the fine mesh is only three time finer than the coarse mesh. The lower and upper bounds for the coarse mesh $(N=12)$ calculations were 0.0069617 and 0.0079325 , respectively. This compares to 0.006425 and 0.009708 for the current method. The previous hierarchical bounds are three times shaper with a half bound gap of $6.5 \%$. Nevertheless these bounds do not provide the full certainty that the exact bounds procedure provides.

\begin{tabular}{|c|c|c|c|c|c|}
\hline \hline$N$ & $h$ & $s_{h}^{-}$ & $s_{h}$ & $s_{h}^{\text {pre }}$ & $s_{h}^{+}$ \\
\hline 3 & 0.333333 & 0.002616 & 0.002616 & 0.011605 & 0.020594 \\
6 & 0.166667 & 0.005199 & 0.005199 & 0.008990 & 0.012781 \\
12 & 0.083333 & 0.006425 & 0.006425 & 0.008067 & 0.009708 \\
18 & 0.055556 & 0.006770 & 0.006770 & 0.007799 & 0.008828 \\
24 & 0.041667 & 0.006921 & 0.006921 & 0.007679 & 0.008437 \\
30 & 0.033333 & 0.007004 & 0.007004 & 0.007613 & 0.008221 \\
36 & 0.027778 & 0.007056 & 0.007056 & 0.007572 & 0.008087 \\
\hline \hline
\end{tabular}

Table 2: The exact Stokes' output bounds obtained on mesh $h$.

Figure 9a) shows the ratios of lower bound, upper bound and discrete output to our most accurate numerical output value $\left(s_{h}^{-} / \tilde{s}, s_{h} / \tilde{s}\right.$ and $\left.s_{h}^{+} / \tilde{s}\right)$ with mesh size $h$. Note that the lower bounds output value is the same of the finite element output value because the forcing term in the Stokes equations and the output functional are constants.

Figure 9b) shows the convergence rate for each bound. Note that we assume that $\tilde{s}$ has a negligent difference from the exact solution, $s$, and is used for the convergence rate estimations: $e_{h}^{-}=\left|s_{h}^{-}-\tilde{s}\right|, e_{h}=\left|s_{h}-\tilde{s}\right|$, and $e_{h}^{+}=\left|s_{h}^{+}-\tilde{s}\right|$. The corresponding convergence rates of are 1.22, 1.22 and 1.13, respectively. The convergence rates are consistent with the prediction of the Stokes bounds method.

The effectivity of the bounds [24] indicates the sharpness by comparing the error in the upper 
and lower bounds to the error in the finite element approximation: $\Theta_{h}^{-}=\frac{\left|\tilde{s}-s_{h}^{-}\right|}{\left|\tilde{s}-s_{h}\right|}$ and $\Theta_{h}^{+}=\frac{\left|\tilde{s}-s_{h}^{+}\right|}{\left|\tilde{s}-s_{h}\right|}$. Table 3 shows that the effectivities of the upper bounds obtained on different mesh sizes are about 2.9 to 3.5 , indicating that the exact bounds converge at the same rate as the finite element output value. These values show that the bounds are sharp as they are just three times the error of the finite element output error. Note that in this case the bounds for the Stokes output are behaving better that for the Poisson output. We also define predicted finite element output solution, $s_{h}^{\text {pre }}$, by the average of the lower and upper bounds $s_{h}^{p r e}=\left(s_{h}^{+}+s_{h}^{-}\right) / 2$ and an error effectivity index as: $\tau_{h}^{\text {pre }}=\frac{\tilde{s}-s_{h}^{\text {pre }}}{\tilde{s}-s_{h}}$. This index indicates that the average of bounds is not doing better or worst than the approximation of the output by the finite element method but the bounds do provide the desired certainty on the exact output. We define yet another effectivity index as $\tau_{h}^{ \pm}=\frac{s_{h}^{ \pm}-s_{h}}{\tilde{s}-s_{h}}$ to measure the quality of the error estimator $s_{h}^{ \pm}-s_{h}$ as compared to the error $\tilde{s}-s_{h}$. For the output considered here, the effectivity of the upper bound is close to 1.3 which indicates that the finite element output on each mesh is about half way between the upper bound and the exact output values. The effectivity of the lower bound is 0 which indicates that the finite element output value is a lower bound.

\begin{tabular}{|c|c|c|c|c|c|c|}
\hline \hline$N$ & $h$ & $\Theta_{h}^{+}$ & $\Theta_{h}^{-}$ & $\tau_{h}$ & $\tau_{h}^{+}$ & $\tau_{h}^{-}$ \\
\hline 3 & 0.333333 & 2.85 & 1.0 & -0.93 & -1.35 & 0 \\
6 & 0.166667 & 2.64 & 1.0 & -0.82 & -1.38 & 0 \\
12 & 0.083333 & 2.83 & 1.0 & -0.91 & -1.35 & 0 \\
18 & 0.055556 & 3.01 & 1.0 & -1.01 & -1.33 & 0 \\
24 & 0.0416667 & 3.19 & 1.0 & -1.09 & -1.31 & 0 \\
30 & 0.0333333 & 3.36 & 1.0 & -1.18 & -1.30 & 0 \\
36 & 0.0277778 & 3.54 & 1.0 & -1.27 & -1.28 & 0 \\
\hline \hline
\end{tabular}

Table 3: Effectivity of bounds.

a)

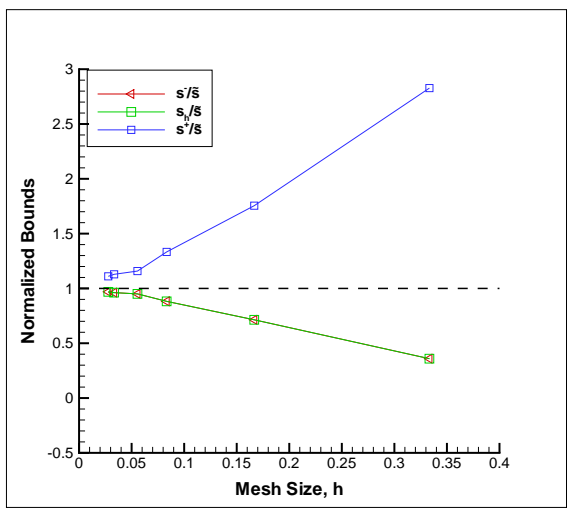

b)

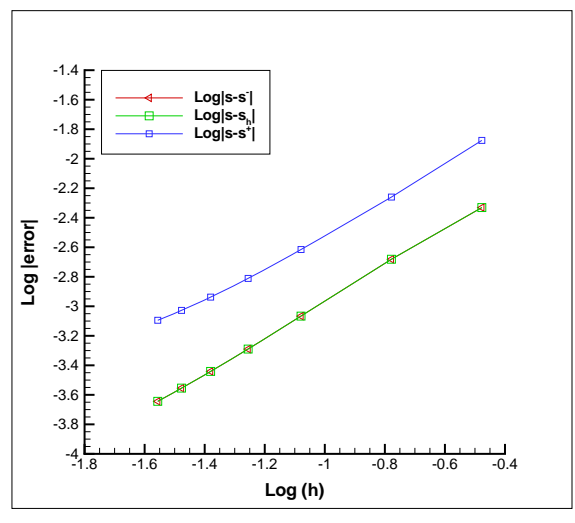

Figure 9: Bounds for Stokes' output (a) upper and lower bounds (b) convergence of the bounds.

\section{Conclusion}

In this paper, we present a new A Posteriori Finite Element Exact Bounds Method for the computation of guaranteed upper and lower bounds for the linear functional outputs of the exact weak solutions to the three dimensional Stokes equations. The bounds method is based on a domain 
decomposition finite element technique exploiting the augmented Lagrangian bounded by a complimentary energy functional. The stationarity condition of the reformulated Lagrangian leads to the global and hybrid flux calculations on a low-order polynomial finite element space and to the decoupled error estimation calculations in each subdomain on a high-order polynomial space. Bounds on the flowrate for a flow over an array of square cylinders are calculated. We report that these bounds are rigorous and provide a certificate of precision for a predicted output with full certainty.

This methods is nevertheless restricted to computational domains with piecewise straight boundaries to avoid accounting for the geometrical error arising from the finite disctretization of curved boundaries. A method could be developed so that the error contribution to the output for curved geometries is calculated and added to the existing bounds. In our model problem the surfaces are aligned with the coordinate system though this is not a restriction of the method. The extension of this method to bound outputs of the Navier-Stokes equation will require a linearization of the Navier-Stokes equations as in [5] which will lead to exact bounds only when a fine enough mesh is used in the calculations. These bounds are then called asymptotic bounds. A similar technique should be used for nonlinear output functional. Clearly, for engineering practice these extensions need to be developed.

\section{Acknowledgements}

This work was supported in part by the Natural Sciences and Engineering Research Council of Canada (NSERC) and Fonds Québéc de la Recherche sur la Nature et les Technologies (FQRNT).

\section{References}

[1] Babuška, I, Strouboulis, T, The Finite Element Method and its Reliability. Oxford University Press: Oxford, 2001.

[2] Babuška, I, Miller, A., "The post-processing approach in the finite element method-Part 3: A posteriori error estimates and adaptive mesh selection", International Journal for Numerical Methods in Engineering, v. 20, p. 2311-2324, 1984.

[3] Bertrand,F. H., Gadbois, M.R., Tanguy,P.A., "Tetrahedral elements for fluid flow", International Journal for Numerical Methods in Engineering, v. 33, p. 1251-1267, 1992.

[4] Chamoin, L., Ladevèze, P., "Bounds on history-dependent or independent local quantities in viscoelasticity problem solved by approximate methods", International Journal for Numerical Methods in Engineering, published online.

[5] Cheng, Z., Paraschivoiu, M., " A posteriori finite element bounds to linear functional outputs of the three-dimensional Navier-Stokes equations", International Journal for Numerical Methods in Engineering, v. 61(11), p. 1835-1859, 2004.

[6] Cheng, Z." A posteriori finite element bounds: application to outputs of the three-dimensional Navier-Stokes equations", Ph.D. Thesis, University of Toronto, 2003.

[7] Choi, H., Paraschivoiu, M., "Adaptive computations of a posteriori finite element output bounds: a comparison of the "hybrid-flux" approach and the "flux-free" approach", Comp. Meth. Appl. Engrg.,v. 193, p. 4001-4033, 2004. 
[8] Choi H., Paraschivoiu, M., "A faster ""hybrid-flux"" calculation approach to output bounds for three dimensional problems", International Journal of Computational fluid Dynamics, V. 19(2), p. 115-130, 2005.

[9] Crouzeix, M., Raviart, P.-A. "Conforming and nonconforming finite element methods for solving the stationary Stokes equations", RAIRO Ser. Rouge v. 7 p. 33-75, 1973.

[10] Díez, P., Calderón, G., "Goal-oriented error estimation for transient parabolic problems", Notas de matematica, v. 2(246), 2006.

[11] Ladevèze, P., Leguillon, D., "Error estimation procedures in the finite element method and applications",SIAM J. Numer. Anal., v. 20, p. 485-509 1983.

[12] Ladevèze, P., Pelle J-P. Mastering Calculations in the Linear and Nonlinear Mechanics. Springer:New York, 2004.

[13] Melbø, H., Kvamsdal, T., "Goal oriented error estimators for the Stokes equations based on variationally consistent postprocessing", Comp. Meth. Appl. Mech. Engrg., v. 192, p. 613-633, 2003.

[14] Oden, J.T., Prudhomme, S., "Goal-Oriented Error Estimation and Adaptivity for the Finite Element Method",Computers and Mathematics with Applications, v. 41, p. 735-756, 2001.

[15] Oden, J.T., Prudhomme, S., Demkowicz, L., "A Posteriori Error Estimation for Acoustic Wave Propagation Problems", Archives of Computation Methods in Engineering, v. 12(4), 2005.

[16] Oden, J.T., Prudhomme, S. "New Approaches to Error Estimation and Adaptivity for the Stokes and Oseen Equations", International Journal for Numerical Methods in Fluids, v. 31, p 3-15, 1999.

[17] Paraschivoiu, M., "A Posteriori Finite Element Output Bounds in Three Space Dimensions Using the FETI Method",Comput. Methods Appl. Mech. Engrg, v. 190, p. 6629-6640, 2001.

[18] Paraschivoiu, M., Patera, A.T., "A hierarchical duality approach to bounds for the outputs of partial differential equations", Comput. Methods Appl. Mech. Engrg, v. 158, p. 389-407, 1998.

[19] Paraschivoiu, M., Peraire, J, and Patera, A.T., "A posteriori finite element bounds for linearfunctional outputs of elliptic partial differential equations",Comput. Methods Appl. Mech. Engrg, v. 150, p. 289-312, 1997.

[20] Paraschivoiu, M., and Patera, A.T., "A posteriori bounds for linear-functional outputs of Crouzeix-Raviart finite element discretization of the incopressible Stokes problem", International Journal for Numerical Methods in Fluids, v. 32(7), p. 823-849, 2000.

[21] Pares, N., Bonet, J., Huerta, A. and Peraire, J., "The Computation of Bounds for LinearFunctional Outputs of Weak Solutions to the Two-Dimensional Elasticity Equations", Comput. Methods Appl. Mech. Engrg, v. 195(4-6), p. 406-429, 2006.

[22] Pares, N., Diez, P., Huerta, A., "Subdomain-Based flux-free a posteriori error estimators", Comput. Methods Appl. Mech. Engrg, v. 195(4-6), p. 297-323, 2006.

[23] Prudhomme, S., Oden, J.T., "On goal-oriented error estimation for elliptic problems: application to the control of pointwise errors",Comp. Meth. Appl. Mech. Engrg., v. 176, p. 313-331, 1999. 
[24] Sauer-Budge, A. M., Bonet, J., Huerta, A., and Peraire, J., "Computing Bounds for Linear Functionals of Exact Weak Solutions to Poisson's Equation", SIAM Journal on Numerical Analysis, v. 42(4), p. 1610-1630, 2004.

[25] Sauer-Budge, A. M., Peraire, J., "Computing Bounds for Linear Functionals of Exact Weak Solutions to the Advection-Diffusion-Reaction Equation",SIAM Journal on Scientific Computing, v. 26, issue 2, p. 636-652, 2004. 


\section{A Augmented Lagrangian construction}

The construction of an augmented Lagrangian is presented in this appendix. A quadratic "energy" based on error functions is reformulated for the desired output of the three dimensional Stokes equations. The constraints in the new Lagrangian include the Stokes finite element equilibrium equations and the inter-subdomain continuity requirements for the three dimensional space.

\section{A.1 General residuals}

To construct the augmented Lagrangian, let $\mathbf{e}=\mathbf{u}-\overline{\mathbf{u}}, \epsilon_{h}=p-\bar{p}$.

Noting that $\mathbf{e}=\mathbf{u}-\overline{\mathbf{u}} \in X$ and $\epsilon=p-\bar{p} \in \mathcal{Q}$, equations (34) and (35) lead to the following equations:

$$
\begin{aligned}
a(\overline{\mathbf{u}}, \mathbf{e})+b(\mathbf{e}, p) & =\ell^{N}(\mathbf{e}), \\
b(\overline{\mathbf{u}}, \epsilon) & =0 .
\end{aligned}
$$

Rewriting the above equations lead to:

$$
\begin{aligned}
a(\mathbf{e}, \mathbf{e})+b(\mathbf{e}, \epsilon) & =\ell^{N}(\mathbf{e})-[a(\overline{\mathbf{u}}, \mathbf{e})+b(\mathbf{e}, \bar{p})], \\
b(\mathbf{e}, \epsilon) & =-[b(\overline{\mathbf{u}}, \epsilon)] .
\end{aligned}
$$

The two right hand sides of Equations (105) and (106) suggest the general residuals to be defined as:

$$
\begin{array}{llrl}
\ell_{1}^{E}(\mathbf{v}) & \equiv \ell^{N}(\mathbf{v})-[a(\overline{\mathbf{u}}, \mathbf{v})+b(\mathbf{v}, \bar{p})], & \forall \mathbf{v} \in \hat{X} ; \\
\ell_{2}^{E}(\beta) & \equiv-[b(\overline{\mathbf{u}}, \beta)], & \forall \beta \in \mathcal{Q} .
\end{array}
$$

Thus, the energy equality can be written as:

$$
a(\mathbf{e}, \mathbf{e})+2 b(\mathbf{e}, \epsilon)-\ell_{1}^{E}(\mathbf{e})-\ell_{2}^{E}(\epsilon)
$$

The specific choice of $\overline{\mathbf{u}}=\mathbf{u}$ and $\bar{p}=p$ lead to $\mathbf{e}=0$ and $\epsilon=0$ and the energy equality vanishes.

\section{A.2 Quadratic-linear Lagrangian}

Now introduce the set of functions $\mathcal{S} \subset \hat{X} \times \mathcal{Q}$,

$$
\mathcal{S}=\left\{\begin{array}{c|cccc}
\mathbf{v} \in \hat{X} & a(\overline{\mathbf{u}}+\mathbf{v}, \mathbf{w})+b(\mathbf{w}, \bar{p}+\beta) & = & \ell^{N}(\mathbf{w}), & \forall \mathbf{w} \in X ; \\
\beta \in \mathcal{Q} & b(\overline{\mathbf{u}}+\mathbf{v}, r) & = & 0, & \forall r \in \mathcal{Q} \\
& H(\mathbf{v}, \mu) & = & 0, & \forall \mu \in \mathbf{E} .
\end{array}\right.
$$

The third constraint $H(\mathbf{v}, \mu)=0, \forall \mu \in \mathbf{E}$ enforces velocity continuity and the homogeneous essential conditions. Therefore, the space of functions $\mathcal{S}$ consists of two functions, e and $\epsilon$. The following trivial minimization statement can be written:

$$
\pm s_{h}^{N S}=\inf _{\mathbf{v} \in \mathcal{S}} \sup _{\beta \in \mathcal{S}} \kappa\left\{a(\mathbf{v}, v)+2 b(\mathbf{v}, q)-\left[\ell_{1}^{E}(\mathbf{v})+\ell_{2}^{E}(\beta)\right]\right\} \pm \ell^{0}(\overline{\mathbf{u}}+\mathbf{v}),
$$

where $\kappa \in \Re_{+}$is a non-negative parameter that will be used to optimize the computed bounds.

The constrained minimization problem suggests the formation of the quadratic-linear Lagrangian $\mathcal{L}: \hat{X} \times \mathcal{Q} \times X \times \mathcal{Q} \times \mathbf{E} \rightarrow \Re$,

$$
\begin{aligned}
\mathcal{L}^{ \pm}(\mathbf{v}, \beta, \mathbf{w}, r, \mu)= & \kappa\left\{a(\mathbf{v}, v)+2 b(\mathbf{v}, \beta)-\left[\ell_{1}^{E}(\mathbf{v})+\ell_{2}^{E}(\beta)\right]\right\} \pm \ell^{0}(\overline{\mathbf{u}}+\mathbf{v}) \\
& +\left\{a(\overline{\mathbf{u}}+\mathbf{v}, \mathbf{w})-\ell^{N}(\mathbf{w})+b(\mathbf{w}, \bar{p}+\beta)+b(\overline{\mathbf{u}}+\mathbf{v}, r)\right\} \\
& +H(\mathbf{v}, \mu),
\end{aligned}
$$


where the first line represents the quadratic "energy" reformation of the desired output, the second line represents the original Stokes problem constrained by the Lagrangian multiplies $\mathbf{w}$ and $r$, and the third line represents the hybrid flux constraints that "glue" the subdomains together.

Equation (109) can also be written as:

$$
\begin{aligned}
& \mathcal{L}^{ \pm}(\mathbf{v}, \beta, \mathbf{w}, r, \mu) \\
& =\kappa\left\{a(\mathbf{v}, v)+2 b(\mathbf{v}, \beta)-\ell^{N}(\mathbf{v})+a(\overline{\mathbf{u}}, \mathbf{v})+b(\mathbf{v}, \bar{p})+b(\overline{\mathbf{u}}, \beta)\right\} \pm \ell^{0}(\overline{\mathbf{u}}+\mathbf{v}) \\
& +\left\{a(\overline{\mathbf{u}}+\mathbf{v}, \mathbf{w})-\ell^{N}(\mathbf{w})+b(\mathbf{w}, \bar{p}+\beta)+b(\overline{\mathbf{u}}+\mathbf{v}, r)\right\}+H(\mathbf{v}, \mu)
\end{aligned}
$$

which leads to

$$
\pm s_{h}^{N S}=\inf _{\mathbf{v} \in \hat{X}} \sup _{\beta \in \mathcal{Q}, \mathbf{w} \in X, r \in \mathcal{Q}, \mu \in \mathbf{E}} \mathcal{L}^{ \pm}(\mathbf{v}, \beta, \mathbf{w}, r, \mu) .
$$




\section{B Calculation of candidate Lagrange multipliers}

Prior to calculating the bounds to the output of interest, the Lagrange multiplier candidates, adjoint and hybrid flux, are required and are calculated on the mesh $\mathcal{T}_{h}$.

\section{B.1 Low order adjoint calculation}

To find the saddle point of the augmented Lagrangian (108) in the subspaces $\hat{X}_{h}^{\text {low }} \subset \hat{X}, \mathcal{Q}_{h} \in$ $\mathcal{Q}, \mathbf{E}_{h}^{\text {low }} \subset \mathbf{E}$, the functions denoted by $\mathbf{v}=\mathbf{e}_{h}^{ \pm}+\nu, \beta=\epsilon_{h}^{ \pm}, \mathbf{w}=\psi_{h}^{ \pm}, r=\theta_{h}^{ \pm}$and $\mu=\mathbf{t}_{h}^{ \pm}$are substituted into the Lagrangian for the specific choice of $\overline{\mathbf{u}}=\mathbf{u}_{h}$ and $\bar{p}=p_{h}$ :

$$
\begin{aligned}
& \mathcal{L}^{ \pm}\left(\mathbf{e}_{h}^{ \pm}+\nu, \epsilon_{h}^{ \pm}, \psi_{h}^{ \pm}, \theta_{h}^{ \pm}, \mathbf{t}_{h}^{ \pm}\right) \\
& =\mathcal{L}^{ \pm}\left(\mathbf{e}_{h}^{ \pm}, \epsilon_{h}^{ \pm}, \psi_{h}^{ \pm}, \theta_{h}^{ \pm}, \mathbf{t}_{h}^{ \pm}\right)+\kappa a(\nu, \nu) \\
& +\kappa\left\{2 a\left(\mathbf{e}_{h}^{ \pm}, \nu\right)+2 b\left(\nu, \epsilon_{H}^{ \pm}\right)-\ell^{N}(\nu)+a\left(\mathbf{u}_{h}, \nu\right)+b\left(\nu, p_{h}\right)\right\} \pm \ell^{0}(\nu) \\
& +\left\{a\left(\nu, \psi_{h}^{ \pm}\right)+b\left(\nu, \theta_{h}^{ \pm}\right)\right\}+H\left(\nu, t_{h}^{ \pm}\right) .
\end{aligned}
$$

Thus, the variation of $\mathcal{L}^{ \pm}$with respect to $\mathbf{v}$ vanishes leads to

$$
\begin{aligned}
& \kappa\left\{2 a\left(\mathbf{e}_{h}^{ \pm}, \mathbf{w}\right)+2 b\left(\mathbf{w}, \epsilon_{h}^{ \pm}\right)-\ell^{N}(\mathbf{w})+a\left(\mathbf{u}_{h}, \mathbf{w}\right)+b\left(\mathbf{w}, p_{h}\right)\right\} \pm \ell^{0}(\mathbf{w}) \\
& +\left\{a\left(\mathbf{w}, \psi_{h}^{ \pm}\right)+b\left(\mathbf{w}, \theta_{h}^{ \pm}\right)\right\}+H\left(\mathbf{w}, t_{h}^{ \pm}\right)=0, \quad \forall \mathbf{w} \in \hat{X}_{h}^{l o w} .
\end{aligned}
$$

Similarly, the stationarity conditions are obtained by requiring that other variations of $\mathcal{L}^{ \pm}$ with respect to $\beta, \mathbf{w}, r$ and $\mu$ vanish.

Therefore, the stationarity conditions are: Find $\mathbf{e}_{h}^{ \pm} \in \hat{X}_{h}^{\text {low }}, \epsilon_{h}^{ \pm} \in \mathcal{Q}_{h}, \psi_{h}^{ \pm} \in X_{h}^{\text {low }}, \theta_{h}^{ \pm} \in \mathcal{Q}_{h}^{\text {low }}$, and $\mathbf{t}_{h}^{ \pm} \in \mathbf{E}_{h}^{\text {low }}$ such that

$$
\begin{aligned}
& \kappa\left\{2 a\left(\mathbf{e}_{h}^{ \pm}, \mathbf{w}\right)+2 b\left(\mathbf{w}, \epsilon_{h}^{ \pm}\right)-\ell^{N}(\mathbf{w})+a\left(\mathbf{u}_{h}, \mathbf{w}\right)+b\left(\mathbf{w}, p_{h}\right)\right\} \pm \ell^{0}(\mathbf{w}) \\
& \quad+a\left(\mathbf{w}, \psi_{h}^{ \pm}\right)+b\left(\mathbf{w}, \theta_{h}^{ \pm}\right)+H\left(\mathbf{w}, \mathbf{t}_{h}^{ \pm}\right)=0, \quad \forall \mathbf{w} \in \hat{X}_{h}^{\text {low }}, \\
& \kappa\left[2 b\left(\mathbf{e}_{h}^{ \pm}, \beta\right)+b\left(\mathbf{u}_{h}, \beta\right)\right]+b\left(\psi_{h}^{ \pm}, \beta\right)=0, \quad \forall \beta \in \mathcal{Q}_{h}, \\
& H\left(\mathbf{e}_{h}^{ \pm}, \mu\right)=0, \quad \forall \mu \in \mathbf{E}_{h}^{\text {low }}, \\
& a\left(\mathbf{u}_{h}+\mathbf{e}_{h}^{ \pm}, \mathbf{w}\right)-\ell^{N}(\mathbf{w})+b\left(\mathbf{w}, p_{h}+\epsilon_{h}^{ \pm}\right)=0, \quad \forall \mathbf{w} \in X_{h}^{\text {low }}, \\
& b\left(\mathbf{u}_{h}, r\right)+b\left(\mathbf{e}_{h}^{ \pm}, r\right)=0, \quad \forall r \in \mathcal{Q}_{h} .
\end{aligned}
$$

Replacing $\mathbf{e}_{h}^{ \pm}$and $\epsilon_{h}^{ \pm}$for the specific choice of $\overline{\mathbf{u}}=\mathbf{u}_{h}$ and $\bar{p}=p_{h}$ into (109) and (110) gives:

$$
\begin{aligned}
a(\mathbf{u}, \mathbf{w})+b(\mathbf{w}, p) & =\ell^{N}(\mathbf{w}), \quad \forall \mathbf{w} \in X_{h}^{\text {low }}, \\
b(\mathbf{u}, \beta) & =0, \quad \forall \beta \in \mathcal{Q}_{h} .
\end{aligned}
$$

These two equations are trivial from (34) and (35).

Substituting Equations (42),(43), (111) and (112) into (106) and (107) yields

$$
\begin{aligned}
a\left(\mathbf{w}, \psi_{h}^{ \pm}\right)+b\left(\mathbf{w}, \theta_{h}^{ \pm}\right) \pm \ell^{0}(\mathbf{w})+H\left(\mathbf{w}, \mathbf{t}_{h}^{ \pm}\right) & =0, \quad \forall \mathbf{w} \in \hat{X}_{h}^{\text {low }}, \\
b\left(\psi_{h}^{ \pm}, \beta\right) & =0, \quad \forall \beta \in \mathcal{Q}_{h} .
\end{aligned}
$$

By constraining w to the continuous $X_{h}^{\text {low }}$ the term $H\left(\mathbf{w}, \mathbf{t}_{h}^{ \pm}\right)$is eliminated in (113) because $X_{h}^{\text {low }}$ is continuous and therefore has no inter-subdomain jumps. Finally Equations (113) and (114) are simplified as

$$
\begin{aligned}
a\left(\mathbf{w}, \psi_{h}^{ \pm}\right)+b\left(\mathbf{w}, \theta_{H}^{ \pm}\right) \pm \ell^{0}(\mathbf{w}) & =0, \quad \forall \mathbf{w} \in X_{h}^{\text {low }} \\
b\left(\psi_{h}^{ \pm}, \beta\right) & =0, \quad \forall \beta \in \mathcal{Q}_{h} .
\end{aligned}
$$


The adjoint can be evaluated as $\psi_{h}^{ \pm}= \pm \psi_{h}$ and $\theta_{h}^{ \pm}= \pm \theta_{h}$. Thus, the above equations about the adjoint calculation are independent of stabilization parameter $\kappa$.

$$
\begin{aligned}
a\left(\mathbf{w}, \psi_{h}\right)+b\left(\mathbf{w}, \theta_{h}\right) & =-\ell^{0}(\mathbf{w}), \quad \forall \mathbf{w} \in X_{h}^{\text {low }}, \\
b\left(\psi_{h}, \beta\right) & =0, \quad \forall \beta \in \mathcal{Q}_{h} .
\end{aligned}
$$

\section{B.2 Low order hybrid flux calculation}

Here, the hybrid flux vector is considered. Let $\mathbf{e}_{h}^{ \pm}=\mathbf{e}_{h}^{p r} \pm \frac{1}{\kappa} \mathbf{e}_{h}^{d u}, \epsilon_{h}^{ \pm}=\epsilon_{h}^{p r} \pm \frac{1}{\kappa} \epsilon_{h}^{d u}$, and $\mathbf{t}_{h}^{ \pm}=$ $\kappa \mathbf{t}_{h}^{p r} \pm \mathbf{t}_{h}^{d u}$. Substituting these parameters into (106) and (107) leads to Equations (117) and (118), respectively,

$$
\begin{aligned}
& \kappa\left\{2 a\left(\mathbf{e}_{h}^{p r}, \mathbf{w}\right)+2 b\left(\mathbf{w}, \epsilon_{h}^{p r}\right)\right\}+\kappa\left\{a\left(\mathbf{u}_{h}, \mathbf{w}\right)-\ell^{N}(\mathbf{w})+b\left(\mathbf{w}, p_{h}\right)\right\} \\
& \quad \pm\left\{2 a\left(\mathbf{e}_{h}^{d u}, \mathbf{w}\right)+2 b\left(\mathbf{w}, \epsilon_{h}^{d u}\right)\right\} \pm \ell^{0}(\mathbf{w}) \pm a\left(\mathbf{w}, \psi_{H}\right) \pm b\left(\mathbf{w}, \theta_{h}\right) \\
& \quad+\kappa H\left(\mathbf{w}, t_{H}^{p r}\right) \pm H\left(\mathbf{w}, t_{h}^{d u}\right)=0, \quad \forall \mathbf{w} \in \hat{X}_{h}^{l o w}, \\
& \kappa\left\{2 b\left(\mathbf{e}_{h}^{p r}, \beta\right)+b\left(\mathbf{u}_{h}, \beta\right)\right\} \pm\left\{2 b\left(\mathbf{e}_{h}^{d u}, \beta\right)+b\left(\psi_{h}, \beta\right)\right\}=0, \quad \forall \beta \in \mathcal{Q}_{h} .
\end{aligned}
$$

Therefore, the hybrid flux calculations can still be separated into two parts that are independent of $\kappa$. The first part is related to the residual of the primal problem, or the original problem on the "broken space": Find $\mathbf{t}_{h}^{p r} \in \mathbf{E}_{h}^{\text {low }}$ such that

$$
\begin{array}{rlrl}
2 a\left(\mathbf{e}_{h}^{p r}, \mathbf{w}\right)+2 b\left(\mathbf{w}, \epsilon_{h}^{p r}\right)+H\left(\mathbf{w}, \mathbf{t}_{h}^{p r}\right) & =\ell^{N}(\mathbf{w})-a\left(\mathbf{u}_{h}, \mathbf{w}\right)-b\left(\mathbf{w}, p_{h}\right), \\
2 b\left(\mathbf{e}_{h}^{p r}, \beta\right) & =-b\left(\mathbf{u}_{h}, \beta\right)=0, & \forall \beta \in \mathcal{Q}_{h}, \\
H\left(\mathbf{e}_{h}^{p r}, \mu\right) & =0, & \forall \mu \in \mathbf{E}_{h}^{\text {low }} .
\end{array}
$$

The hybrid flux for the dual problem is: Find $\mathbf{t}_{h}^{d u} \in \mathbf{E}_{h}^{\text {low }}$, such that

$$
\begin{array}{rlrl}
2 a\left(\mathbf{e}_{h}^{d u}, \mathbf{w}\right)+2 b\left(\mathbf{w}, \epsilon_{h}^{d u}\right)+H\left(\mathbf{w}, t_{h}^{d u}\right) & =-\ell^{0}(\mathbf{w})-a\left(\mathbf{w}, \psi_{h}\right)-b\left(\mathbf{w}, \theta_{h}\right), \\
& \forall \mathbf{w} \in \hat{X}_{h}^{\text {low }}, \\
2 b\left(\mathbf{e}_{h}^{d u}, \beta\right) & =-b\left(\psi_{h}, \beta\right)=0, & \forall \beta \in \mathcal{Q}_{h}, \\
H\left(\mathbf{e}_{H}^{d u}, \mu\right) & =0, & \forall \mu \in \mathbf{E}_{h}^{l o w} .
\end{array}
$$

The directly equilibrated hybrid-flux approach is applied to solve the above dual interface problems. 